\title{
Abnormal Cortico-Basal Ganglia Neurotransmission in a Mouse Model of L-DOPA-Induced Dyskinesia
}

\author{
${ }^{\circledR}$ Indriani Dwi Wahyu, ${ }^{1,2}{ }^{\circledR}$ Satomi Chiken, ${ }^{1,2}$ Taku Hasegawa, ${ }^{1}{ }^{\circledR}$ Hiromi Sano, ${ }^{1,2}$ and ${ }^{\circledR}$ Atsushi Nambu ${ }^{1,2}$ \\ ${ }^{1}$ Division of System Neurophysiology, National Institute for Physiological Sciences, Okazaki 444-8585, Japan, and ${ }^{2}$ Department of Physiological \\ Sciences, SOKENDAI (Graduate University for Advanced Studies), Okazaki 444-8585, Japan
}

L-3,4-dihydroxyphenylalanine (L-DOPA) is an effective treatment for Parkinson's disease (PD); however, long-term treatment induces L-DOPA-induced dyskinesia (LID). To elucidate its pathophysiology, we developed a mouse model of LID by daily administration of L-DOPA to PD male ICR mice treated with 6-hydroxydopamine (6-OHDA), and recorded the spontaneous and cortically evoked neuronal activity in the external segment of the globus pallidus (GPe) and substantia nigra pars reticulata (SNr), the connecting and output nuclei of the basal ganglia, respectively, in awake conditions. Spontaneous firing rates of GPe neurons were decreased in the dyskinesia-off state ( $\geq 24 \mathrm{~h}$ after L-DOPA injection) and increased in the dyskinesia-on state (20-100 min after L-DOPA injection while showing dyskinesia), while those of $\mathrm{SNr}$ neurons showed no significant changes. GPe and $\mathrm{SNr}$ neurons showed bursting activity and low-frequency oscillation in the PD, dyskinesia-off, and dyskinesia-on states. In the GPe, cortically evoked late excitation was increased in the PD and dyskinesia-off states but decreased in the dyskinesia-on state. In the $\mathrm{SNr}$, cortically evoked inhibition was largely suppressed, and monophasic excitation became dominant in the PD state. Chronic L-DOPA treatment partially recovered inhibition and suppressed late excitation in the dyskinesia-off state. In the dyskinesia-on state, inhibition was further enhanced, and late excitation was largely suppressed. Cortically evoked inhibition and late excitation in the $\mathrm{SNr}$ are mediated by the cortico-striato-SNr direct and cortico-striato-GPe-subthalamo-SNr indirect pathways, respectively. Thus, in the dyskinesia-on state, signals through the direct pathway that release movements are enhanced, while signals through the indirect pathway that stop movements are suppressed, underlying LID.

Key words: basal ganglia; direct and indirect pathways; external segment of the globus pallidus; extracellular recording; L-DOPA-induced dyskinesia; substantia nigra pars reticulata

Significance Statement

Parkinson's disease (PD) is caused by progressive loss of midbrain dopaminergic neurons, characterized by tremor, rigidity, and akinesia, and estimated to affect around six million people world-wide. Dopamine replacement therapy is the gold standard for PD treatment; however, control of symptoms using L-3,4-dihydroxyphenylalanine (L-DOPA) becomes difficult over time because of abnormal involuntary movements (AIMs) known as L-DOPA-induced dyskinesia (LID), one of the major issues for advanced PD. Our electrophysiological data suggest that dynamic changes in the basal ganglia circuitry underlie LID; signals through the direct pathway that release movements are enhanced, while signals through the indirect pathway that stop movements are suppressed. These results will provide the rationale for the development of more effective treatments for LID.

Received Feb. 4, 2020; revised 0ct. 16, 2020; accepted Dec. 11, 2020.

Author contributions: I.D.W., S.C., H.S., and A.N. designed research; I.D.W., and H.S. performed research;

I.D.W., S.C., T.H., and H.S. analyzed data; I.D.W., S.C., T.H., H.S., and A.N. wrote the paper.

This work was supported by Grants-in-Aid for Scientific Research "Non-linear Neuro-oscillology" $15 \mathrm{H} 05873$ (to A.N.) and "Adaptive Circuit Shift" $15 \mathrm{H} 01458$ and $17 \mathrm{H} 05590$ (to H.S.) from the Ministry of Education, Culture, Sports, Science and Technology, Japan; Grants-in-Aid for Scientific Research 16 K01968 (to H.S.), 16K07014 (to S.C.), and 26250009 (to A.N.) from the Japan Society for the Promotion of Science; and Core Research for Evolutional Science and Technology Grant JPMJCR1853 (to S.C.) from Japan Science and Technology Agency. We thank S. Sato, N. Suzuki, K. Awamura, K. Miyamoto, M. Goto, H. Isogai, and

T. Sugiyama for their technical support and $Y$. Yamagata for her critical reading of the manuscript.

The authors declare no competing financial interests.

Correspondence should be addressed to Atsushi Nambu at nambu@nips.ac.jp or Hiromi Sano at hsano@nips.ac.jp.

https://doi.org/10.1523/JNEUROSCI.0267-20.2020

Copyright $\odot 2021$ the authors

\section{Introduction}

Parkinson's disease $(\mathrm{PD})$ is a neurodegenerative disorder caused by progressive loss of dopaminergic neurons in the substantia nigra pars compacta $(\mathrm{SNc})$ and characterized by tremor, rigidity, akinesia, and non-motor symptoms (Albin et al., 1989; DeLong, 1990; Fahn, 2003). Administration of L-3,4-dihydroxyphenylalanine (L-DOPA) compensates for reduced dopamine and ameliorates PD symptoms (Cotzias et al., 1969; Fahn, 2003). However, disease progression and long-term L-DOPA treatment induce motor fluctuations and dyskinesia in the majority of PD patients within 10 years, and make the control of PD symptoms difficult (Ahlskog and Muenter, 2001; Cenci, 2014). L-DOPA-induced dyskinesia (LID) characterized by choreiform and dystonic 
movements (Fahn, 2000; Obeso et al., 2000a; Bezard et al., 2001; Cenci and Lundblad, 2006) limits the quality of life of PD patients, and its control is a major issue for advanced PD. Once LID appears, it occurs in every L-DOPA administration (Cenci and Lundblad, 2006; Jenner, 2008), suggesting latent and irreversible changes after long-term L-DOPA treatment.

Molecular and biochemical changes have been reported in LID (Cenci, 2007, 2014), including excessive release of dopamine from serotonergic terminals, supersensitivity of postsynaptic dopaminergic receptors, changes of intracellular signaling in striatal (Str) neurons, and abnormal cortico (Cx)-Str plasticity (Picconi et al., 2003, 2011). Synaptic connectivity and dendritic morphology of Str projection neurons were also altered (Fieblinger et al., 2014; Nishijima et al., 2014; Suárez et al., 2014, 2016). On the other hand, studies at the circuit level have been rather limited. Recent imaging studies showed that LID induced differential activity changes in Str direct-pathway $\left(\mathrm{Str}_{\mathrm{d}}\right)$ and indirect-pathway (Str $\left.{ }_{i}\right)$ neurons (Parker et al., 2018; Ryan et al., 2018), and their selective optogenetic/chemogenetic activation exacerbated or alleviated LID (Alcacer et al., 2017; Hernández et al., 2017; Perez et al., 2017; Girasole et al., 2018; Ryan et al., 2018; Keifman et al., 2019).

To elucidate the pathophysiology of LID systematically at the circuit level, here we recorded neuronal activity in the external segment of the globus pallidus (GPe) and substantia nigra pars reticulata $(\mathrm{SNr})$ using a mouse model of LID in awake conditions. The GPe is the connecting nucleus of the basal ganglia (BG), while the SNr together with the internal segment of the globus pallidus (GPi) is the output nucleus (Albin et al., 1989; Alexander and Crutcher, 1990; DeLong, 1990). Their activity was altered in animal models and human patients of movement disorders (Filion and Tremblay, 1991; Hutchinson et al., 1997; Boraud et al., 1998, 2001; Wichmann et al., 1999; Lozano et al., 2000; Obeso et al., 2000b; Levy et al., 2001; Heimer et al., 2002, 2006; Wichmann and Soares, 2006).

We paid special attention to their responses to motor $\mathrm{Cx}$ stimulation, a triphasic response composed of early excitation, inhibition, and late excitation in the GPe and SNr/GPi in the normal state. Each component is mediated by the Cx-subthalamo (STN)-GPe/SNr/GPi, Cx-Str-GPe/SNr/GPi, and Cx-StrGPe-STN-GPe/SNr/GPi pathways, respectively based on the following findings: (1) blockade of the STN suppressed both early excitation and late excitation in the GPe/SNr/GPi; (2) local $\mathrm{GABA}_{\mathrm{A}}$ receptor blockade diminished inhibition in the GPe/ GPi; (3) ablation of $\mathrm{Str}_{\mathrm{i}}$-GPe neurons diminished inhibition and late excitation in the GPe and late excitation in the $\mathrm{SNr}$ (Ryan and Clark, 1991; Maurice et al., 1999; Nambu et al., 2000, 2002; Kita et al., 2004; Tachibana et al., 2008; Sano et al., 2013). Response pattern changes in the GPe and SNr/GPi reflect neurotransmission efficacy through the BG circuitry. Moreover, in voluntary movements, activity originating in the $\mathrm{Cx}$ is transmitted through the BG circuitry and reaches the SNr/GPi. Thus, Cx stimulation can mimic such information processing through the BG. Indeed, $\mathrm{Cx}$-evoked response patterns are altered in movement disorders and could explain their pathophysiology (Nambu et al., 2000; Chiken et al., 2008, 2015; Kita and Kita, 2011; Nishibayashi et al., 2011; Sano and Nambu, 2019). To reveal latent changes after chronic L-DOPA treatment, we also examined the dyskinesia-off state when the effect of L-DOPA was washed out, and LID was not observed.

\section{Materials and Methods}

\section{Animals}

Twelve adult male ICR mice (SLC; $\sim 10$ weeks old, $36-42 \mathrm{~g}$ before the operation) were used. The experimental procedures were approved by the Institutional Animal Care and Use Committee of National Institutes of Natural Sciences. All experiments were conducted according to the National Institutes of Health Guide for Care and Use of Laboratory Animals. Mice were housed under a 12/12 h light/dark cycle, with free access to water and food. Before experiments, mice were handled daily to become accustomed to the experimenters.

\section{Experimental design}

Animals were separated into two groups (Fig. 1A). The vehicle (shamlesioned group, six mice) or 6-hydroxydopamine (6-OHDA; 6-OHDAlesioned group, six mice) was injected unilaterally to the medial forebrain bundle (MFB) of the right hemisphere (Fig. 1A, day -14). Fourteen days after vehicle/6-OHDA injection (day 0), the first cylinder test was conducted to evaluate PD motor features. The first surgery to mount a head holder on the mouse's head $(\mathrm{day}-3)$ and the second surgery to chronically implant stimulating electrodes in the motor $\mathrm{Cx}$ (day 3 ) were performed $3 \mathrm{~d}$ before and $3 \mathrm{~d}$ after the cylinder test, respectively. Then, spontaneous activity and $\mathrm{Cx}$-evoked responses of $\mathrm{GPe}$ and $\mathrm{SNr}$ neurons were recorded in the sham-lesioned ("control" state) and 6OHDA-lesioned ("PD" state) groups (from day 11 to 42 ) in the awake state. After recording in the control or PD state, all mice received daily injections of L-DOPA $(10 \mathrm{mg} / \mathrm{kg}$, i.p.) for $11 \mathrm{~d}$ (from day 42 to 52 ). Abnormal involuntary movements (AIMs) were examined before and after L-DOPA treatment every other day. The second cylinder test was performed to evaluate PD motor features after the termination of chronic L-DOPA treatment (day 53). Then, recordings of the activity of GPe and SNr neurons were restarted in both the sham-lesioned ("LDOPA-off/on" states) and 6-OHDA-lesioned ("dyskinesia-off/on" states) groups (from day 56 to 95) along with acute L-DOPA treatment $(10 \mathrm{mg} / \mathrm{kg}$, i.p.). When recordings were not performed, L-DOPA treatment continued two to four times per week to maintain AIMs scores. After the final recording (day 100), animals were perfused, and recording sites in the GPe and $\mathrm{SNr}$ and dopaminergic neurons in the $\mathrm{SNc}$ were examined histologically.

\section{Vehicle or 6-OHDA injection}

6-OHDA was injected into the MFB according to standard methods (Francardo et al., 2011; Thiele et al., 2011; Fig. 1A, day -14); $30 \mathrm{~min}$ before vehicle or 6-OHDA injection, desipramine hydrochloride (25 $\mathrm{mg} / \mathrm{kg}$, i.p., norepinephrine reuptake inhibitor; Sigma-Aldrich) was administered to mice to increase the selectivity of 6-OHDA-induced lesions. Vehicle $(0.02 \%$ ascorbic acid dissolved in saline) or 6-OHDAhydrobromide $(3 \mathrm{mg} / \mathrm{ml}$ in $0.02 \%$ ascorbic acid dissolved in saline; Sigma-Aldrich) was prepared immediately before surgeries. Each mouse was anesthetized with ketamine hydrochloride $(100 \mathrm{mg} / \mathrm{kg}$, i.p.) and xylazine hydrochloride $(5 \mathrm{mg} / \mathrm{kg}$, i.p.) and held in the stereotaxic apparatus. The skin of the head was incised, and a small hole was made on the right side of the skull to access the MFB. A glass micropipette was connected to a Hamilton microsyringe, and was inserted into the right MFB (posterior $1.2 \mathrm{~mm}$, lateral $1.3 \mathrm{~mm}$, and ventral $4.75 \mathrm{~mm}$ from bregma; Francardo et al., 2011) according to the mouse brain atlas (Franklin and Paxinos, 2008). The vehicle or 6-OHDA solution was then injected with a microinfusion pump ( $1 \mu \mathrm{l}$ at $0.2 \mu \mathrm{l} / \mathrm{min}$; Micro4, WPI). After injection, the glass micropipette was left in place for $5 \mathrm{~min}$ to allow diffusion away from the injection site, and then the micropipette was slowly retracted. The incised skin was closed with surgical clips (Reflex Clip, WPI).

\section{First surgery for head fixation}

Three days before the first cylinder test (Fig. 1A, day -3), the surgical operation to mount a head holder on the mouse's head was performed as described previously (Chiken et al., 2008; Sano et al., 2013). Each mouse was anesthetized with ketamine hydrochloride $(100 \mathrm{mg} / \mathrm{kg}$, i.p.) and xylazine hydrochloride $(5 \mathrm{mg} / \mathrm{kg}$, i.p.) and held in the stereotaxic apparatus. The skull was widely exposed and covered with bone adhesive resin (Bistite II, Tokuyama Dental). A small U-shape head holder made 


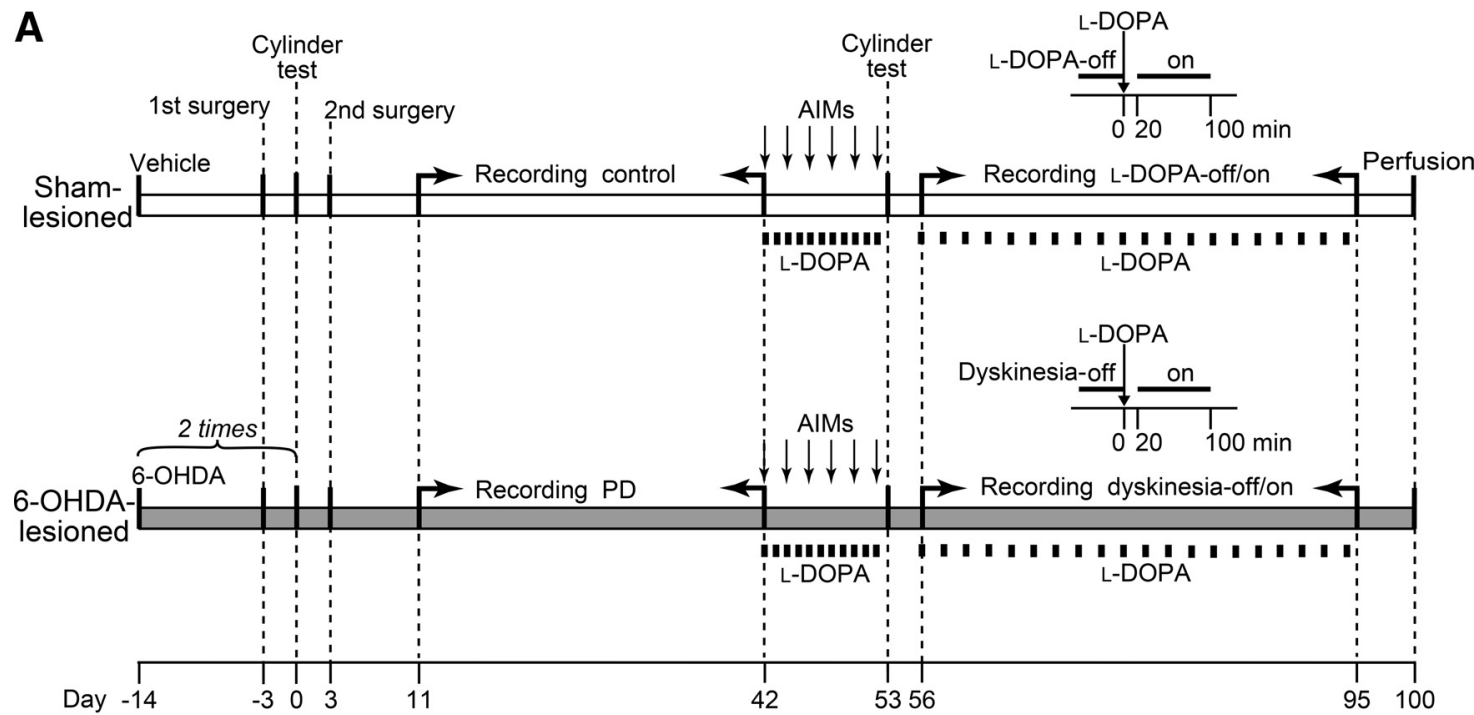

B

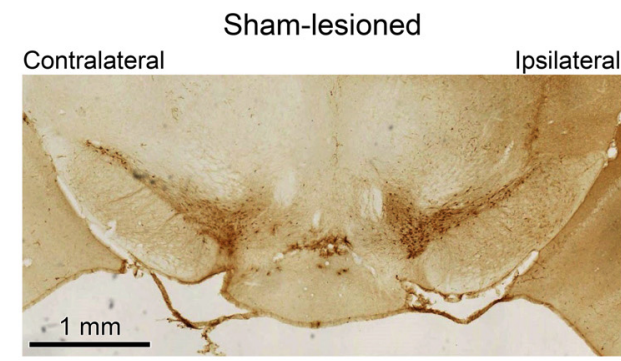

C

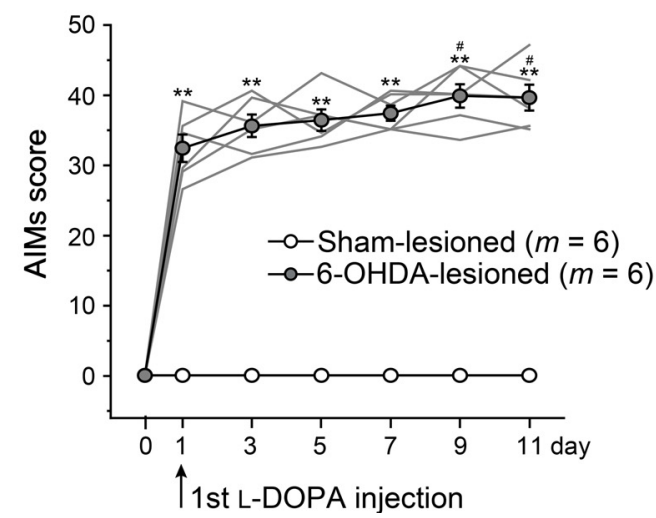

6-OHDA-lesioned
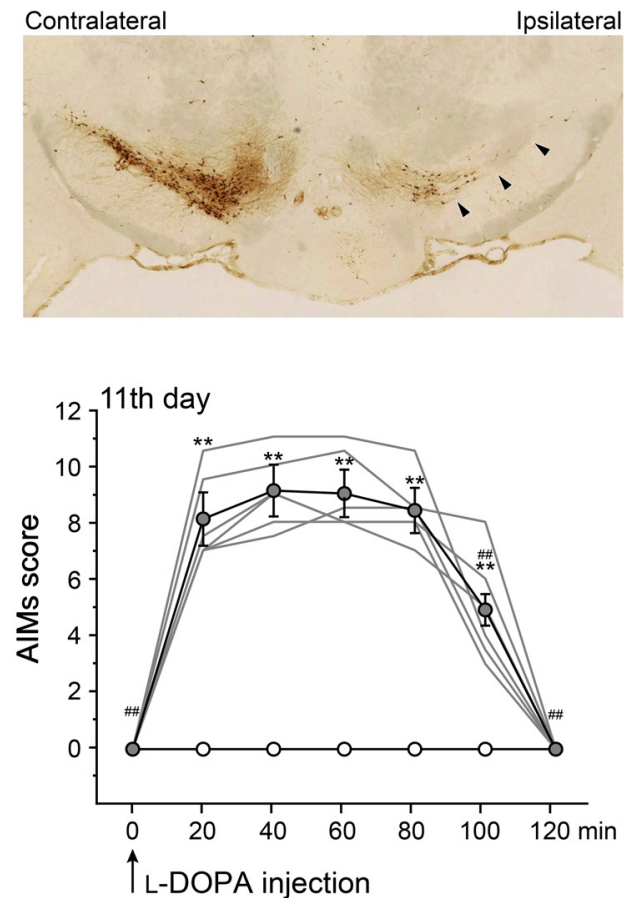

Figure 1. Experimental design, histologic verification, and behavioral evaluation. $\boldsymbol{A}$, The experimental timeline depicts vehicle or 6-OHDA injection into the MFB of two groups of mice (sham lesioned and 6-OHDA lesioned), surgeries for head fixation and extracellular recordings, cylinder tests, the first extracellular recordings in the control and PD states, L-DOPA treatments, AlMs test, and the second extracellular recordings in the sham-lesioned and 6-OHDA-lesioned groups. The second recording session was divided into the L-DOPA-off/dyskinesia-off ( $\geq 24 \mathrm{~h}$ after the previous L-DOPA treatment and before the next L-DOPA treatment) and L-DOPA-on/dyskinesia-on states (from 20 to 100 min after acute L-DOPA injection; inset images above the recording information). Cylinder test was performed on days 0 and 53 . AlMs test was conducted once every other day as indicated by vertical lines with arrows between days 42 and 52 . $\boldsymbol{B}$, Immunohistological staining with TH to visualize dopaminergic neurons in the SNc. Vehicle (left) or 6-OHDA (right) was injected into the ipsilateral MFB. Dopaminergic neurons in the 6-OHDA injection side were markedly reduced in number (arrowheads). C, AIMs scores during chronic L-DOPA treatment for the evaluation of LID. Left, L-DOPA was injected every day for $11 \mathrm{~d}$, and total AIMs scores were measured every other day and one day before L-DOPA treatment as a control; ${ }^{* *} p<0.001$ significantly different from the 0 th day, $\# p<0.05$ significantly different from the first day, two-way repeated-measures ANOVA with Tukey's post hoc test. Right, Total AIMs scores during $120 \mathrm{~min}$ after the L-DOPA injection on the 11 th day; ${ }^{* *} p<0.001$ significantly different from time $0 \mathrm{~min}, \# \# p<0.001$ significantly different from time 20 to $80 \mathrm{~min}$. Mean $\pm \mathrm{SE}$ (black lines) and individual mouse scores (gray lines) are shown. $m$, number of mice.

of acetal resin was attached to the skull with acrylic resin (Unifast II, GC).

Cylinder test to evaluate PD motor features

Fourteen days after vehicle or 6-OHDA injection (Fig. $1 A$, day 0), PD motor features were evaluated using the cylinder test (Schallert et al., 2000; Tillerson et al., 2001; Meredith and Kang, 2006). Each mouse was placed inside a transparent acrylic cylinder (inner diameter $10 \mathrm{~cm}$, height $20 \mathrm{~cm}$ ) and videotaped for $10 \mathrm{~min}$ without previous habituation to the cylinder. Two mirrors were placed side-by-side, forming a $90^{\circ}$ angle with each other, behind the cylinder to allow views from all sides of the cylinder. Mice showed exploratory behavior by rearing and leaning on the wall of the cylinder with their forelimbs. The numbers of rotations $\left(\geq 180^{\circ}\right)$ during $10 \mathrm{~min}$ and of contacts with their right or left forelimb 
for the first 5 min were counted (Cenci and Lundblad, 2007; Francardo et al., 2011; Alcacer et al., 2017). A contralateral forelimb use score was calculated with the number of wall contacts by the left forelimb (contralateral to the lesion, affected side) as a percentage of the total number of wall contacts by the right or left forelimb. If mice showed no impairment in the usage of the left forelimb compared with that of the right forelimb (contralateral forelimb use score $\geq 20 \%$ ), additional injections of 6-OHDA were performed. In the present study, all mice received 6-OHDA injections twice and showed significant impairment. Another cylinder test was performed after chronic L-DOPA treatment (day 53) to examine its long-term therapeutic effects on PD motor features.

\section{Second surgery for implantation of stimulating electrodes}

Three days after the first cylinder test (Fig. $1 A$, day 3 ), the second surgery was performed to chronically implant stimulating electrodes in the motor Cx. The mouse was anesthetized with ketamine hydrochloride $(50-100 \mathrm{mg} / \mathrm{kg}$, i.p.) and then held in the stereotaxic apparatus with its head restrained using the U-shape head holder. Part of the skull in the right hemisphere, ipsilateral to the vehicle/6-OHDA injection side, was removed to access the motor $\mathrm{Cx}, \mathrm{GPe}$, and SNr. Two pairs of bipolar stimulating electrodes $(50-\mu \mathrm{m}$ diameter Teflon-coated tungsten wires, tip distance $300-400 \mu \mathrm{m}$ ) were implanted chronically into the orofacial and forelimb regions of the primary motor $\mathrm{Cx}$, and fixed using acrylic resin (Chiken et al., 2008; Sano et al., 2013). Somatotopy of these regions was confirmed by intracortical microstimulation (a train of 10 pulses at $333 \mathrm{~Hz}, 200-\mu$ s duration, $\leq 35 \mu \mathrm{A})$.

\section{Neuronal activity recording in the control or PD state}

After recovery from the second surgery (Fig. $1 A$, from day 11 to 42 ), the awake mouse in the control or PD state was positioned painlessly in a stereotaxic apparatus using the U-shape head holder (Chiken et al., 2008, 2015; Sano et al., 2013). For single-unit recording, a glass-coated tungsten microelectrode ( 0.5 or $1.0 \mathrm{M} \Omega$ at $1 \mathrm{kHz}$; Alpha Omega) was inserted vertically into the GPe (target area; posterior $0.3-0.7 \mathrm{~mm}$ and lateral 1.6-2.4 mm from bregma) or $\mathrm{SNr}$ (posterior $2.9-3.3 \mathrm{~mm}$ and lateral 1.5-1.9 mm from bregma) through the dura mater using a hydraulic microdrive. Signals from the microelectrode were amplified and filtered $(0.3-5.0 \mathrm{kHz})$. Unit activity was isolated, converted to digital data with a homemade time-amplitude window discriminator, and sampled at $2.0 \mathrm{kHz}$ using a computer with LabVIEW 7.1 software (National Instruments) for off-line data analysis. Spontaneous discharges were recorded for $50 \mathrm{~s}$. The responses to Cx-electrical stimulation $(200-\mu$ s duration, monophasic single pulse at $0.7 \mathrm{~Hz}, 50-\mu \mathrm{A}$ strength) through the stimulating electrodes implanted in the motor $\mathrm{Cx}$ were examined by constructing peristimulus time histograms (PSTHs; bin width of $1 \mathrm{~ms}$; prestimulus, $100 \mathrm{~ms}$; poststimulus, $900 \mathrm{~ms}$ ) for 100 stimulation trials.

\section{Chronic L-DOPA treatment}

After finishing the recording session in the control or PD state, shamlesioned and 6-OHDA-lesioned groups were treated daily with L-DOPA for $11 \mathrm{~d}$ (Fig. $1 A$, from day 42 to 52 ). They received an i.p. injection of a mixture of $10 \mathrm{mg} / \mathrm{kg}$ L-DOPA (Dopaston, Ohara Pharmaceutical) and $15 \mathrm{mg} / \mathrm{kg}$ benserazide (peripheral DOPA decarboxylase inhibitor; Sigma-Aldrich) dissolved in saline.

\section{Behavioral test measuring AIMs}

One day before and every other day during chronic L-DOPA treatment (Fig. 1A, from day 42 to 52 ), each mouse was placed inside a transparent acrylic cage (width $26 \mathrm{~cm}$, depth $18 \mathrm{~cm}$, and height $13 \mathrm{~cm}$ ) and videotaped for $1 \mathrm{~min}$ at 20-min intervals during $120 \mathrm{~min}$. LID was assessed by using the AIMs scale described below, which is similar to that used in human dyskinesia (Cenci and Lundblad, 2007; Francardo et al., 2011; Thiele et al., 2011), for $1 \mathrm{~min}$ before (at $0 \mathrm{~min}$ ) and every $20 \mathrm{~min}$ after (at $20,40,60,80,100$, and $120 \mathrm{~min}$ ) L-DOPA injection. Based on the topographical distribution, AIMs were classified into four subtypes: axial (lateral flexion and axial rotation of the neck and trunk toward the side contralateral to the lesioned hemisphere), forelimb (repetitive rhythmic jerky movements or dystonic posturing of the forelimb on the side contralateral to the lesioned hemisphere), orolingual (repetitive and vacuous chewing movements of the jaw with or without tongue protrusion), and locomotive (increased rotational activity toward the contralateral side of the lesion with tactile contacts of at least three paws with the floor). Each subtype was scored on a scale from 0 to 4 ( 0 , absent; 1 , occasional; 2 , frequent; 3 , continuous; 4 , continuous and not interruptible by outer stimuli). Total AIMs scores at each time point were calculated by the sum of AIMs scores in the four different body parts (maximum, 16). Total AIMs scores of the day were calculated by the sum of AIMs scores at each time point after L-DOPA injection (six points in total; maximum, 96).

Neuronal activity recording in the L-DOPA-off/on or dyskinesia-off/on states

After measuring AIMs scores, neuronal activity recording in the L-DOPA-off/on or dyskinesia-off/on states was restarted (Fig. 1A, from day 56 to 95). Spontaneous discharges and the responses to $\mathrm{Cx}$ stimulation were recorded in the GPe and $\mathrm{SNr}$ in awake conditions as described above. Data recorded long ( $\geq 24 \mathrm{~h})$ after the previous L-DOPA treatment and before the next L-DOPA treatment (Fig. $1 A$, inset images) were described as L-DOPA-off (sham-lesioned group) or dyskinesia-off (6-OHDA-lesioned group) when acute L-DOPA effects were washed out, and no AIMs were observed in the 6-OHDA-lesioned group. The dyskinesia-off state corresponds to the latent state of LID after long-term L-DOPA treatment.

After the recording, the awake mouse was kept in the stereotaxic apparatus, and L-DOPA $(10 \mathrm{mg} / \mathrm{kg}$ with benserazide, i.p.) was injected through an elaster needle, which had been inserted into the peritoneal cavity before the experiment. Then, another recording was performed from 20 to $100 \mathrm{~min}$ after acute L-DOPA injection when the 6-OHDAlesioned group showed AIMs (Fig. 1A, inset images). Data recorded during this period were described as L-DOPA-on (sham-lesioned group) or dyskinesia-on (6-OHDA-lesioned group).

\section{Histology}

After the final recording (Fig. 1A, day 100), GPe and SNr recording sites were marked by passing cathodal DC $(20 \mu \mathrm{A}$ for $20 \mathrm{~s})$ through the recording electrode. Mice were then deeply anesthetized with sodium pentobarbital $(100 \mathrm{mg} / \mathrm{kg}$, i.p.) and perfused transcardially with $0.01 \mathrm{~m} \mathrm{PBS}$ followed by $10 \%$ formalin in $0.01 \mathrm{~m}$ PBS. The brains were removed, postfixed in $10 \%$ formalin at $4^{\circ} \mathrm{C}$ overnight and cryoprotected in graded sucrose $(10-30 \%$ sucrose in $0.01 \mathrm{M} \mathrm{PBS})$ at $4^{\circ} \mathrm{C}$. Frontal sections $(40 \mu \mathrm{m})$ were cut with a freezing microtome and collected in $0.01 \mathrm{M}$ PBS as previously described (Sano et al., 2013). Brain sections containing the motor $\mathrm{Cx}, \mathrm{GPe}$, or $\mathrm{SNr}$ were mounted on MAS-coated slide glasses (Matsunami Glass), air-dried, and stained with Neutral Red. The recording sites in the GPe and SNr were confirmed according to the lesions made by cathodal DC and the traces of electrode tracks.

The sections containing the SNc were used to evaluate dopaminergic neurons in the lesioned hemisphere as described previously (Sano et al., 2015). Free-floating sections were incubated with primary antibody against mouse tyrosine hydroxylase (TH; 1:1000; Merck) at $4^{\circ} \mathrm{C}$ overnight, and visualized with biotinylated secondary antibody (1:500; Vector Laboratories) using an $\mathrm{ABC}$ method (Vectastain Elite $\mathrm{ABC}$ kit, Vector Laboratories). The sections were mounted, air-dried, coverslipped, and examined under a light microscope.

\section{Analysis of electrophysiological data}

Spontaneous discharge rates were calculated from continuous digitized recordings for $50 \mathrm{~s}$. Other parameters characterizing firing patterns were calculated from the first $30 \mathrm{~s}$ of the same recordings; the coefficient of variation (CV) of interspike intervals (ISIs), the burst index defined as the ratio of the mean and the mode of ISIs, and the percentage of numbers of spikes in bursts detected by Poisson surprise method [surprise value $\left(-\log _{10} p\right) \geq 2$, the minimum number of spikes during bursts was 3 ; Legéndy and Salcman, 1985; Chiken et al., 2008, 2015; Sano et al., 2013].

Autocorrelograms (bin width of $0.5 \mathrm{~ms}$ ) were calculated from continuous digitized recordings for $50 \mathrm{~s}$ and smoothed with a Gaussian filter $(\sigma=1.6 \mathrm{~ms})$. The mean value and SD were calculated between 0.1 and 
$0.2 \mathrm{~s}$ (200 bins) and considered as the baseline coefficient value. The peaks and troughs were judged significant if the coefficient exceeded the level of mean $\pm 3.29 \mathrm{SD}$ (corresponding to $p=0.001$, two-tailed $t$ test). The firing pattern of the neurons was judged regular if at least one pair of significant peak and trough was observed (Chiken et al., 2008; Tachibana et al., 2008).

The power spectral density (PSD) function was calculated from the same recordings by Welch's method with the Hann window composed of 4096 bins (2048 ms) with 50\% overlapping (Tachibana et al., 2011). The shuffled PSD was also calculated from a shuffled spike train, which was constructed by combining randomly shuffled short segments of the original spike train (one-half of the mean ISIs in length). The original PSD was divided by the shuffled PSD to compensate for the spectral distortion at low frequencies and averaged among neurons.

The responses to Cx stimulation were analyzed by using PSTHs. Cx stimulation typically induced a triphasic response composed early excitation, inhibition, and late excitation in GPe and SNr neurons. The mean value $\left(\mu_{\text {baseline }}\right)$ and $\mathrm{SD}\left(\mathrm{SD}_{\text {baseline }}\right)$ of the discharge rate during $100 \mathrm{~ms}$ preceding the onset of stimulation were considered as the baseline discharge rate, and statistical significance level was set as $\mu_{\text {baseline }} \pm 1.65$ $\mathrm{SD}_{\text {baseline }}$ (corresponding to $p=0.1$, two-tailed $t$ test). If two consecutive bins during 3-7 ms (five bins) for early excitation, three consecutive bins during $5-19$ ms ( 15 bins) for inhibition, and three consecutive bins during $12-42 \mathrm{~ms}$ ( 31 bins) for late excitation exceeded the significance level, the response was judged significant (corresponding to $p=0.04$ for early excitation, $p=0.013$ for inhibition, and $p=0.029$ for late excitation after Bonferroni's correction, two-tailed $t$ test). Once significant bins were detected, the response was considered continuing unless two consecutive bins fell below the significance level (within $\left.\mu_{\text {baseline }} \pm 1.65 \mathrm{SD}_{\text {baseline}}\right)$. The starting and end points were defined as the first and last bins of the response, respectively. In case that early excitation and late excitation were merged without inhibition, the end point of early excitation and the starting point of late excitation were set to 17 and $18 \mathrm{~ms}$, respectively. The duration (from the starting point to the end point) and the amplitude (the area of response; the number of spikes during the significant changes minus the number of spikes during baseline) of each response were calculated and compared in all groups of mice (Sano et al., 2013; Chiken et al., 2015), positive and negative amplitudes represent excitatory and inhibitory responses, respectively. If there was no significant excitation, inhibition, or late excitation, the duration and amplitude were set to zero.

For population PSTHs, the PSTH of each neuron with a significant response to $\mathrm{Cx}$ stimulation was averaged within the same condition and smoothed using a Gaussian filter $(\sigma=1.6 \mathrm{~ms})$. For heat maps, the PSTH of each neuron was standardized using the $\mathrm{Z}$-score $\left[\mathrm{z}_{\mathrm{i}}=\left(\mathrm{y}_{\mathrm{i}}-\mu_{\text {baseline }}\right) /\right.$ $\mathrm{SD}_{\text {baseline, }}$ where $\mathrm{y}_{\mathrm{i}}$ is the firing rate at time $\mathrm{i}$ ], color-coded according to the Z-score, and sorted by the response patterns and the latency of the first component of $\mathrm{Cx}$-evoked responses. Electrophysiological data were analyzed using IgorPro 7 (Wavemetrics), OriginPro 2018 (Lightstone), and MATLAB R2016b (MathWorks) software.

\section{Statistical analysis}

Statistical tests were performed with OriginPro 2018. Behavioral data in the first and second cylinder tests were analyzed by using one-way ANOVA with Tukey's post hoc test. AIMs scores during L-DOPA treatment were analyzed by using two-way repeated-measures ANOVA with Tukey's post hoc test. The parameters of spontaneous neuronal activity and components of $\mathrm{Cx}$-evoked responses were compared by using twoway ANOVA followed by Bonferroni's post hoc test. In case of a significant interaction between two factors (lesion group $\times$ L-DOPA treatment), Kruskal-Wallis test with Dunn's multiple comparison test was used. Proportions of neurons exhibiting regular firings and Cx-evoked response patterns between different states were examined by using $\chi^{2}$ test. All values are expressed as mean \pm SD unless otherwise stated.

\section{Results}

Motor behaviors before and after chronic L-DOPA treatment Vehicle (six mice) or 6-OHDA (six mice) was injected into the right MFB. PD motor features were examined using the cylinder test after $14 \mathrm{~d}$, and 6-OHDA was additionally injected into all 6OHDA-lesioned mice. 6-OHDA-lesioned mice (Fig. 1A, day 0) showed significant ipsilateral rotational behavior (ipsilateral rotation, $10.5 \pm 4.0$ turns; contralateral rotation, 0 turn; $F_{(3,20)}=$ 23.94, $p<0.001$; one-way ANOVA with Tukey's post hoc test), while sham-lesioned mice did not (ipsilateral rotation, $1.5 \pm 1.8$ turns; contralateral rotation, $3.3 \pm 1.5$ turns). 6-OHDA-lesioned mice also showed a significant reduction in the usage of the forelimb contralateral to the lesion in wall contacts (contralateral forelimb use, $\left.16.5 \pm 14.6 \% ; F_{(3,20)}=5.12, p=0.0045\right)$, while all sham-lesioned mice showed similar usage of both forelimbs (contralateral forelimb use, $53.3 \pm 39.4 \%$ ). Histologic examination showed that $\mathrm{TH}$-positive neurons in the SNc were dramatically decreased in 6-OHDA-lesioned mice, but not in shamlesioned mice (Fig. 1B). These behavioral and histologic results confirmed that the 6-OHDA-lesioned mouse could be considered as a PD model.

L-DOPA $(10 \mathrm{mg} / \mathrm{kg}$ ) was administrated daily for $11 \mathrm{~d}$ (Fig. $1 A$, from day 42 to 52), and AIMs of 6-OHDA-lesioned or sham-lesioned mice were examined every other day after L-DOPA treatment (Fig. 1C). Distinct AIMs were observed from the first day of L-DOPA treatment in all 6-OHDA-lesioned mice and continued throughout the period (lesion, $F_{(1,5)}=537.61$, $p<0.001$; time, $F_{(6,30)}=113.57, p<0.001$; lesion $\times$ time, $F_{(6,30)}=113.57, p<0.001 ; p<0.001,1-11 \mathrm{~d}$ in 6-OHDAlesioned mice; two-way repeated-measures ANOVA with Tukey's post hoc test; Fig. $1 C$, left), while no AIMs were observed in sham-lesioned mice $(p>0.99)$. AIMs were observed in all four AIMs subtypes, i.e., axial, forelimb, orolingual, and locomotive dyskinesia. As for the time course of AIMs development within a day, AIMs scores started to increase $20 \mathrm{~min}$ after LDOPA injection, peaked during $40-80 \mathrm{~min}$, and then returned to the control level in $120 \mathrm{~min}$ [11th day of L-DOPA treatment (Fig. $1 C$, right); lesion, $F_{(1,5)}=90.85, p<0.001$; time, $F_{(6,30)}=61.0$, $p<0.001$; lesion $\times$ time, $F_{(6,30)}=61.0, p<0.001 ; p<0.001,20$ $100 \mathrm{~min}$ in 6-OHDA-lesioned mice]. Based on this observation, we defined the dyskinesia-on state as a period between 20 and $100 \mathrm{~min}$ after each L-DOPA injection for the following electrophysiological studies.

The second cylinder test was performed to evaluate PD motor features after chronic L-DOPA treatment (Fig. 1A, day 53) during the dyskinesia-off state. In the 6-OHDA-lesioned mice, abnormal ipsilateral rotational behaviors were reduced (ipsilateral rotation, $3.8 \pm 3.1$ turns; contralateral rotation, $3.5 \pm 1.6$ turns; $\left.F_{(3,20)}=0.68, p=0.99\right)$, while forelimb use asymmetry remained (contralateral forelimb use, $17.3 \pm 24.3 \% ; F_{(3,20)}=5.98, p=0.003$ ). These data suggest that long-term L-DOPA treatment ameliorated some PD motor features such as rotational behaviors.

\section{Spontaneous activity changes of GPe neurons}

Spontaneous firing rates and patterns of GPe neurons were examined in Figure $2 A$. The mean firing rate of GPe neurons (significant interaction between lesion group $\times$ L-DOPA treatment, $F_{(2,589)}=13.86, p<0.001$; two-way ANOVA; Fig. $2 A$ ) was $67.9 \pm 18.2 \mathrm{~Hz}$ in the control state, and it remained unchanged in the L-DOPA-off $(68.2 \pm 21.1 \mathrm{~Hz} ; p>0.99$; Kruskal-Wallis test with Dunn's multiple comparison test) and L-DOPA-on (67.7 \pm $18.0 \mathrm{~Hz} ; p>0.99)$ states. The mean firing rate of GPe neurons in the PD state $(69.3 \pm 18.9 \mathrm{~Hz})$ was not significantly different from that in the control state $(p>0.99)$, whereas that was significantly lower in the dyskinesia-off state $(59.8 \pm 19.3 \mathrm{~Hz})$ and higher in the dyskinesia-on state $(83.8 \pm 23.2 \mathrm{~Hz}$; L-DOPA-off vs dyskinesia-off, $p=0.035$; PD vs dyskinesia-off, $p=0.004$; L-DOPA- 

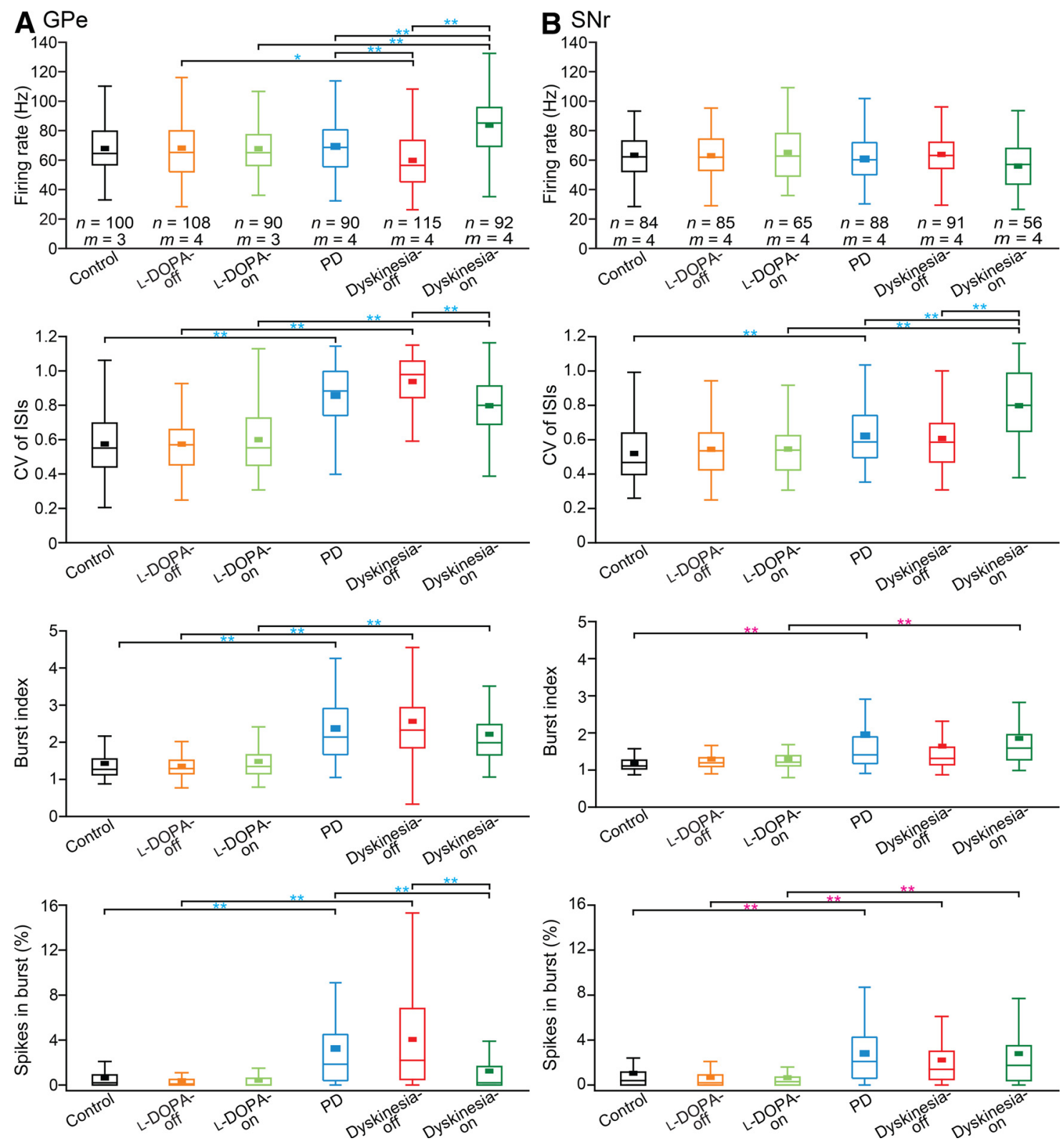

Figure 2. Spontaneous activity in the GPe and SNr. $\boldsymbol{A}, \boldsymbol{B}$, Box plots (the median, first and third quartiles, and minimum and maximum excluding any outliers outside 1.5 times the interquartile range from the upper and lower quartiles) and means (rectangles) of firing rate, CV of ISls, bust index, and spikes in burst of GPe ( $\boldsymbol{A})$ and SNr ( $\boldsymbol{B}$ ) neurons are shown in the control, LDOPA-off, and L-DOPA-on states of the sham-lesioned group and PD, dyskinesia-off, and dyskinesia-on states of the 6-0HDA-lesioned group; $* p<0.05$, $* * p<0.01$ significantly different from each other; cyan *, **two-way ANOVA with Kruskal-Wallis test with Dunn's multiple comparison test; magenta *, **two-way ANOVA with Bonferroni's post hoc test. $n$, number of neurons; $m$, number of mice.

on vs dyskinesia-on, $p<0.001 ; \mathrm{PD}$ vs dyskinesia-on, $p<$ 0.001 ; dyskinesia-off vs dyskinesia-on, $p<0.001)$. The firing patterns were also examined by the $\mathrm{CV}$ of ISIs, the burst index, and the percentage of spikes in bursts (CV of ISIs, significant interaction between lesion group $\times$ L-DOPA treatment, $F_{(2,589)}=11.25, p<0.001$; burst index, $F_{(2,589)}=3.822$, $p=0.022$; spikes in bursts, $F_{(2,589)}=13.86, p<0.001$; Fig. $2 A)$. In the 6-OHDA-lesioned group, the CV of ISIs and bust index were larger in all three states $(p<0.001$; KruskalWallis test with Dunn's multiple comparison test) as compared with the sham-lesioned group, and the percentage of spikes in bursts was larger in the PD and dyskinesia-off states $(p<0.001)$, but not in the dyskinesia-on state $(p=0.189)$.

Typical examples of digitized spikes and smoothed autocorrelograms in each state are shown in Figure 3A. GPe neurons in the control, L-DOPA-off, L-DOPA-on, and dyskinesia-on states fired with weak regularity, while such regularity disappeared in the PD and dyskinesia-off states. The percentage of GPe neurons that exhibited regular firings was much smaller in the PD (9\%) and dyskinesia-off (3\%) states as compared with the sham-lesioned group (control vs PD, $\chi^{2}=30.71, p<0.001$; L-DOPA-off vs dyskinesia-off, $\chi^{2}=63.94, p<0.001 ; \chi^{2}$ test with Bonferroni's correction; Table 1), but not in the dyskinesia-on state (50\%; L-DOPA-on vs dyskinesia-on, $\chi^{2}=2.76$, $p=0.097)$. Strong regularity appeared in the dyskinesia-on state (Fig. 3A), the number of peaks of autocorrelograms (significant effect of lesion group, $F_{(1,183)}=4.13, p<0.043$; two-way ANOVA; Table 1) was larger $(p<0.001$; Bonferroni's post hoc test), and the mean time of the first peak (significant effect of lesion group, $F_{(1,183)}=55.81, p<0.001$; significant effect of 
$\mathrm{AGPe}$

Sham-lesioned

Control
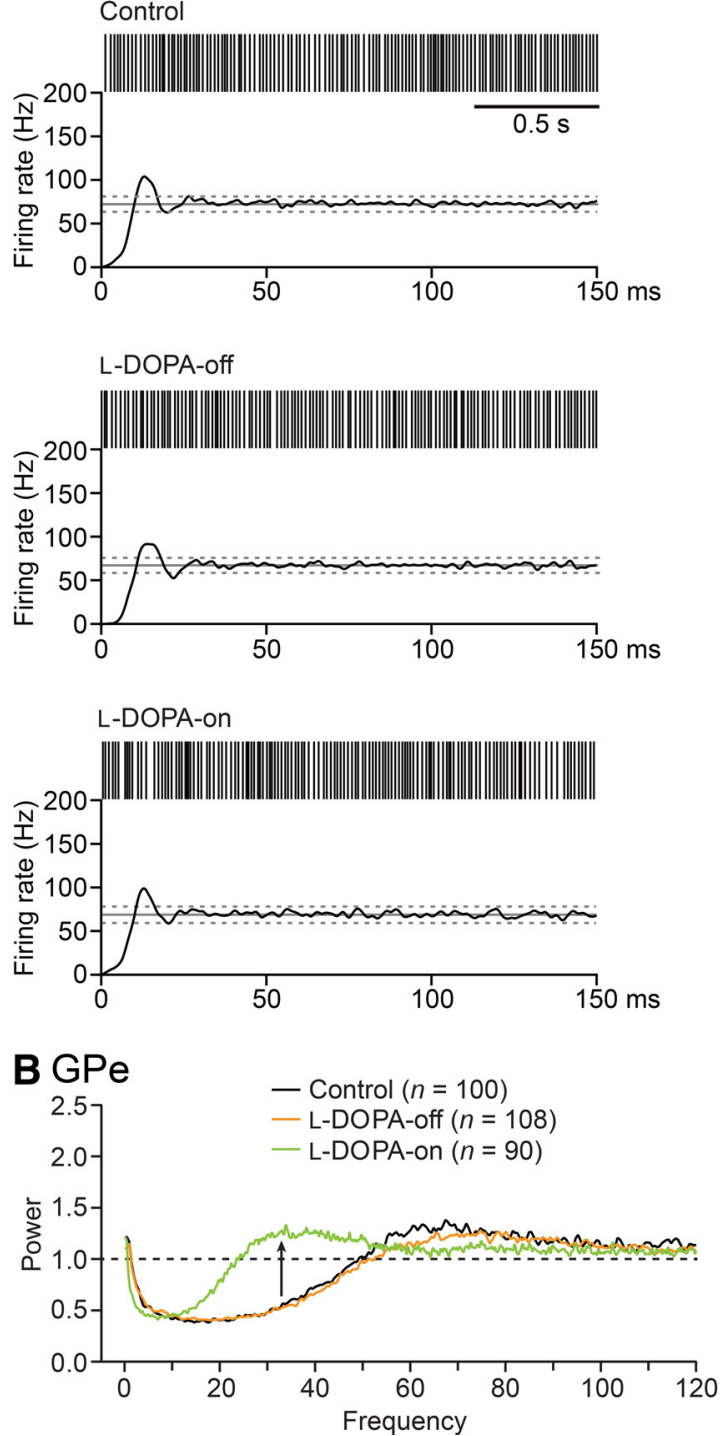
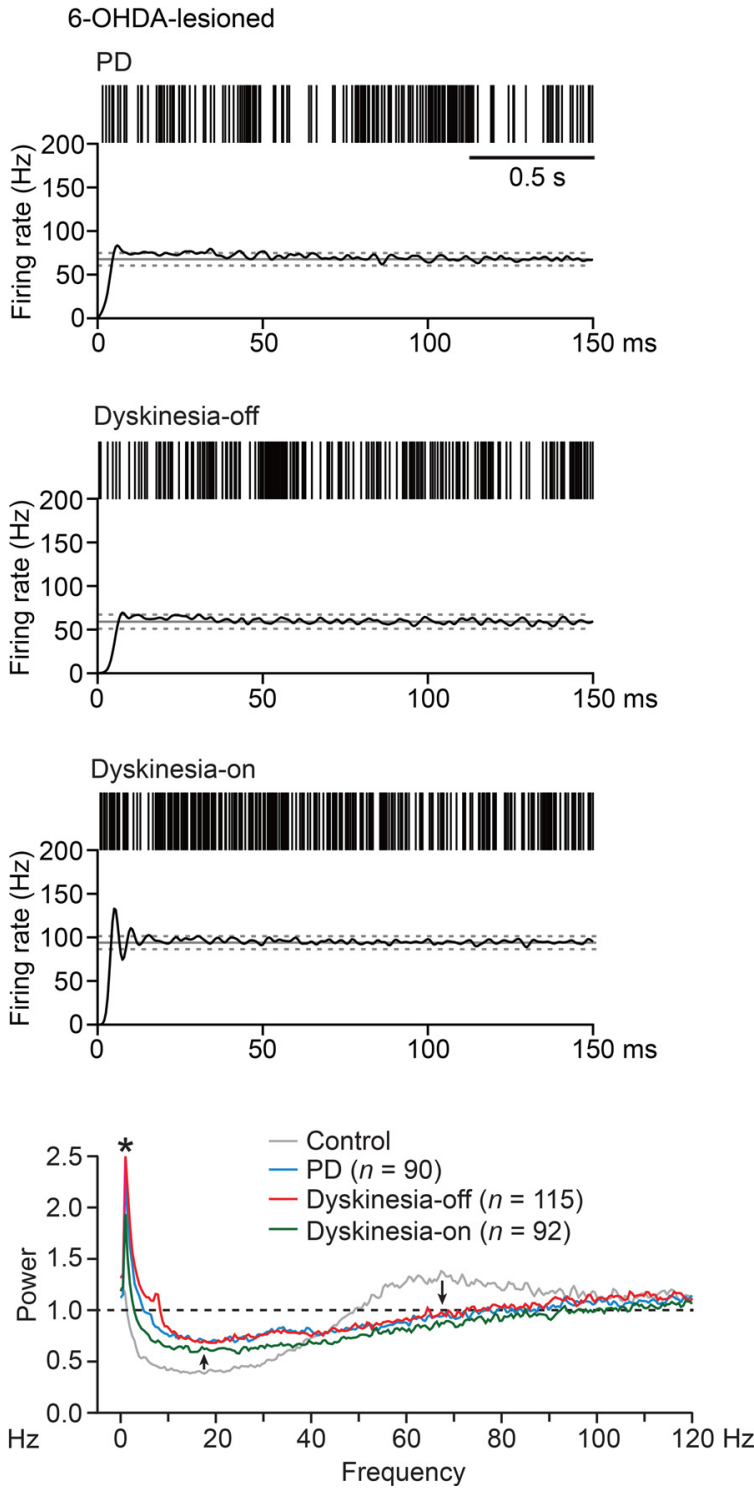

Figure 3. Spontaneous firing patterns of GPe neurons. A, Digitized spikes and smoothed autocorrelograms of spontaneous activity in the control, L-DOPA-off, and L-DOPA-on states of the sham-lesioned group (left), and in the PD, dyskinesia-off, and dyskinesia-on states of the 6-OHDA-lesioned group (right). The mean value (continuous gray line) and $p=0.001$ levels (dashed gray lines) are indicated. $\boldsymbol{B}$, Averaged PSD among neurons in the control, L-DOPA-off, and L-DOPA-on states of the sham-lesioned group (left), and in the PD, dyskinesia-off, and dyskinesia-on states of the 6-OHDA-lesioned group (right). Arrows, activity changes between different states; *peaks around $1 \mathrm{~Hz}$.

Table 1. Regularity of spontaneous activity of GPe and SNr neurons

\begin{tabular}{|c|c|c|c|c|c|c|}
\hline & \multicolumn{3}{|c|}{ Sham lesioned } & \multicolumn{3}{|c|}{ 6-OHDA lesioned } \\
\hline & Control & L-DOPA-off & L-DOPA-on & PD & Dyskinesia-off & Dyskinesia-on \\
\hline \multicolumn{7}{|l|}{ GPe } \\
\hline$m$, number of mice & $m=3$ & $m=4$ & $m=3$ & $m=4$ & $m=4$ & $m=4$ \\
\hline Number of regular-firing neurons (\%) $)^{a}$ & $45(45 \%)$ & $53(49 \%)$ & $34(38 \%)$ & $8(9 \%)^{* *}$ & $3(3 \%)^{* *}$ & $46(50 \%)^{\$ \$}$ \\
\hline Number of peaks of autocorrelograms $s^{b}$ & $1.8 \pm 0.8$ & $1.9 \pm 0.8$ & $1.8 \pm 1.2$ & $2.1 \pm 1.3$ & $2.3 \pm 0.6$ & $3.0 \pm 3.0^{* * *}$ \\
\hline$n$, number of neurons & $n=84$ & $n=85$ & $n=65$ & $n=88$ & $n=91$ & $n=56$ \\
\hline$m$, number of mice & $m=4$ & $m=4$ & $m=4$ & $m=4$ & $m=4$ & $m=4$ \\
\hline Number of regular-firing neurons (\%) $)^{a}$ & $46(55 \%)$ & $49(58 \%)$ & $40(62 \%)$ & $6(7 \%)^{* *}$ & $9(10 \%)^{* * *}$ & $6(11 \%)^{* * *}$ \\
\hline Number of peaks of autocorrelograms $s^{b}$ & $2.1 \pm 0.9$ & $2.1 \pm 0.8$ & $2.1 \pm 1.0$ & $1.5 \pm 0.6$ & $1.4 \pm 0.7$ & $2.0 \pm 1.1$ \\
\hline Time of the 1 st peak $(\mathrm{ms})^{b}$ & $14.8 \pm 3.0$ & $13.7 \pm 3.0$ & $14.5 \pm 3.5$ & $16.0 \pm 4.1$ & $13.9 \pm 2.9$ & $14.2 \pm 3.8$ \\
\hline
\end{tabular}

Values are means $\pm S D, * * p<0.01$ significantly different from the corresponding states of the sham-lesioned group, \#\#p $<0.01$ significantly different from the control state, $\$ \$ p<<0.01$ significantly different from the PD and dyskinesia-off states, ${ }^{a} \chi^{2}$ test with Bonferroni's correction, ${ }^{b}$ two-way ANOVA with Bonferroni's post hoc test. 
A SNr

Sham-lesioned

Control

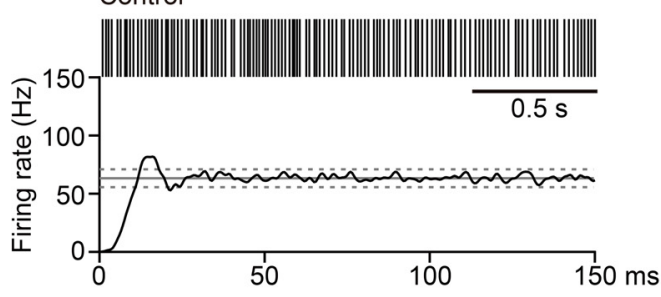

L-DOPA-off

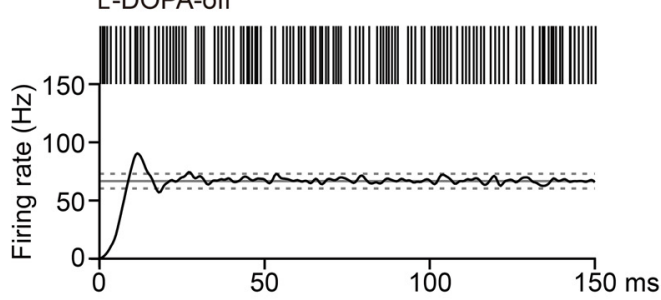

L-DOPA-on
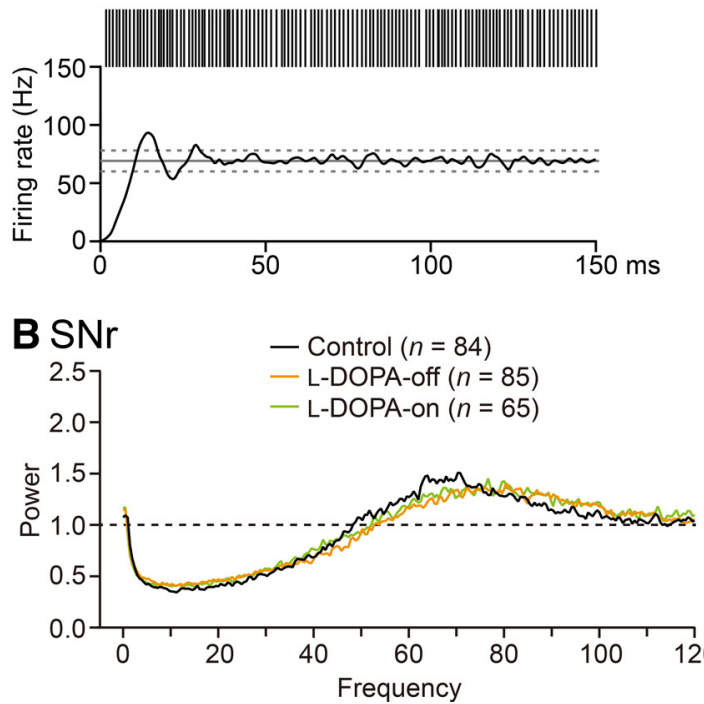

6-OHDA-lesioned
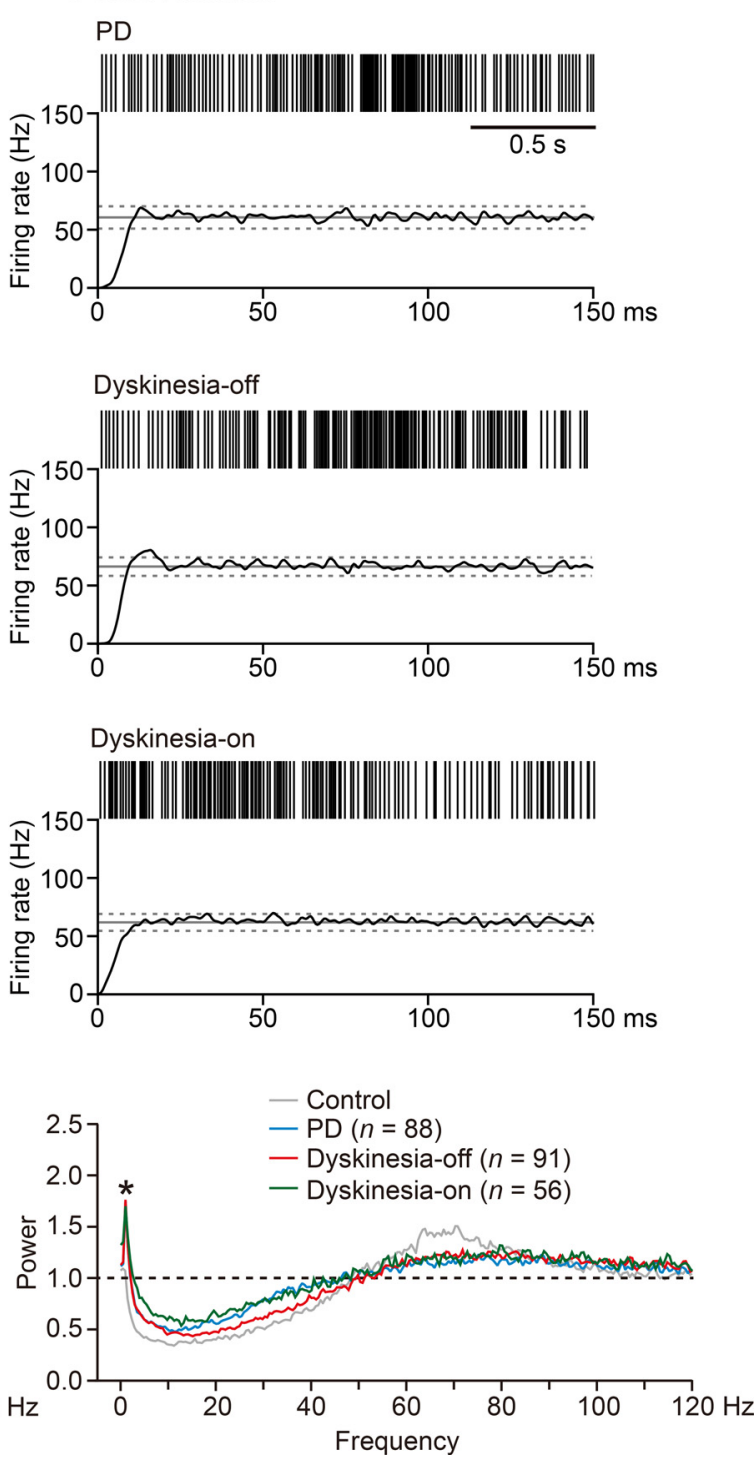

Figure 4. Spontaneous firing patterns of SNr neurons. $\boldsymbol{A}$, Digitized spikes and smoothed autocorrelograms. $\boldsymbol{B}$, Averaged PSD. $*$ peaks around $1 \mathrm{~Hz}$.

L-DOPA treatment, $\left.F_{(2,183)}=5.014, p=0.008\right)$ was shorter $(p<$ $0.001)$, suggesting regular high-frequency firings. The mean time of the first peak was also shorter in the PD state $(p<0.001)$, although the number of neurons with regular firings were small. In the sham-lesioned group, L-DOPA treatment significantly shortened the mean time of the first peak (control vs L-DOPA-off, control vs L-DOPA-on, $p<0.001)$.

Population PSDs of GPe neurons revealed that $60-$ to $80-\mathrm{Hz}$ activity was high, while 10 - to $40-\mathrm{Hz}$ activity was low in the control and L-DOPA-off states (Fig. 3B, left). In the L-DOPA-on state, 10 - to $50-\mathrm{Hz}$ activity was increased (Fig. $3 B$, left, upward arrow). In all three states in the 6-OHDA-lesioned group, 0 - to $40-\mathrm{Hz}$ activity was increased (Fig. $3 B$, right, upward arrow), achieving peaks around $1 \mathrm{~Hz}$ (Fig. $3 B$, right, *), while 50 - to 90 $\mathrm{Hz}$ component was decreased (Fig. $3 B$, right, downward arrow).

\section{Spontaneous activity changes of SNr neurons}

Spontaneous firing rates and patterns of $\mathrm{SNr}$ neurons were examined in Figure $2 B$. The mean firing rates of SNr neurons (significant interaction between lesion group $\times$ L-DOPA treatment,
$F_{(2,463)}=3.844, p=0.022$; two-way ANOVA; Fig. $\left.2 B\right)$ were $63.3 \pm 15.8 \mathrm{~Hz}$ in the control, $63.0 \pm 16.4 \mathrm{~Hz}$ in the L-DOPA-off, and $65.2 \pm 19.3 \mathrm{~Hz}$ in the L-DOPA-on states and remained unchanged in the 6-OHDA-lesioned group $(\mathrm{PD}, 60.8 \pm 15.9 \mathrm{~Hz}$, $p>0.99$; dyskinesia-off, $63.9 \pm 13.8 \mathrm{~Hz}, p>0.99$; dyskinesia-on, $55.9 \pm 16.4 \mathrm{~Hz}, p=0.17$; Kruskal-Wallis test with Dunn's multiple comparison test). The CV of ISIs (significant interaction between lesion group $\times$ L-DOPA treatment, $F_{(2,463)}=10.55, p<0.001$; two-way ANOVA; Fig. $2 B$ ) and burst index (significant effect of lesion group, $F_{(1,463)}=36.64, p<0.001$; Bonferroni's post hoc test) were larger in the PD and dyskinesia-on states as compared with the sham-lesioned group $(p<0.001)$. In addition, the percentages of spikes in bursts (significant effect of lesion group, $F_{(1,463)}=$ $69.00, p<0.001)$ in the 6-OHDA-lesioned group were larger in all three states $(p<0.001)$.

Typical examples of digitized spikes and smoothed autocorrelograms in each state are shown in Figure $4 A$. SNr neurons fired with weak regularity in control, L-DOPA-off, and L-DOPA-on states (Fig. 4A, left), while such regularity disappeared in PD, dyskinesia-off, and dyskinesia-on states. More than $50 \%$ of $\mathrm{SNr}$ 

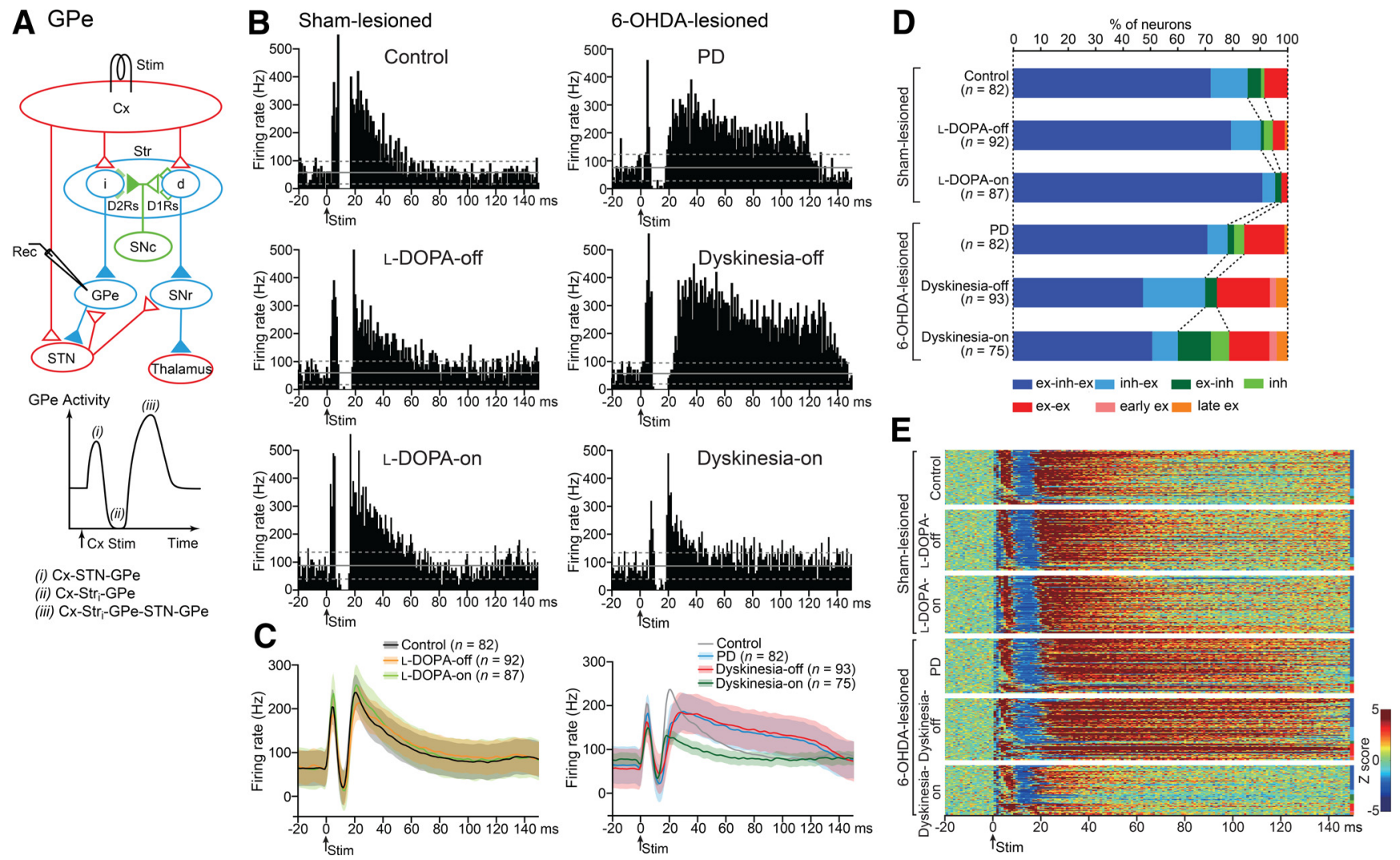

Figure 5. Responses of GPe neurons evoked by motor $(x$ stimulation. $\boldsymbol{A}$, The stimulation $(C x)$ and recording (GPe) sites are depicted with the BG circuitry. Red, cyan, and light-green lines represent glutamatergic excitatory, GABAergic inhibitory, and dopaminergic projections, respectively. In the Str, $\mathrm{d}$ and i represent direct-pathway (Str $\mathrm{d}_{\mathrm{d}}$ ) and indirect-pathway $\left(\mathrm{Str}_{\mathrm{i}}\right)$ neurons, respectively. (x-evoked response in the GPe is typically composed of early excitation (i), inhibition (ii), and following late excitation (iii), which are mediated by the CX-STN-GPe, CX-Stri-GPe, and CX-Stri-GPe-STN-GPe pathways, respectively. B, PSTHs of the typical response of GPe neurons to Cx stimulation in the control, L-DOPA-off, and L-DOPA-on states of the sham-lesioned group (left), and in the PD, dyskinesia-off, and dyskinesia-on states of the 6-OHDA-lesioned group (right). Cx stimulation was delivered at time 0 (arrows) for 100 stimulation trials. The mean value ( $\mu_{\text {baseline}}$ ) and SD (SD baseline) of the discharge rate calculated from the 100-ms period preceding the onset of stimulation, and $\mu_{\text {baseline }}$ (solid gray line) and $\mu_{\text {baseline }} \pm 1.65 \mathrm{SD}_{\text {baseline }}$ (dotted gray lines) are indicated. C, Population PSTHs of (x-evoked responses in GPe neurons of the sham-lesioned (left) and 6-OHDA-lesioned (right) groups. The light-colored areas represent \pm SD, and $n$ indicates the number of neurons used to construct population PSTHs. D, Response patterns of GPe neurons. GPe neurons were classified based on response patterns to $C x$ stimulation in the control, L-DOPA-off, and L-DOPA-on states of the sham-lesioned group (upper), and in the PD, dyskinesia-off, and dyskinesia-on states of the 6-OHDA-lesioned group (lower). Ex, excitation; inh, inhibition. Ex-inh-ex means a triphasic response composed of early excitation, inhibition, and following late excitation. $E$, Heat maps of CX-evoked responses of GPe neurons. The amplitude of responses of GPe neurons was standardized using the Z-score and displayed in the control, L-DOPA-off, and L-DOPA-on states of the sham-lesioned group (upper), and in the PD, dyskinesiaoff, and dyskinesia-on states of the 6-OHDA-lesioned group (lower). Each horizontal line indicates one neuron and was sorted by the response patterns and the latency of the first component of $\mathrm{C}$-evoked responses. The colored bar on the right end of each heat map shows the response patterns as shown in $\boldsymbol{D}$.

neurons exhibited regular firings in the sham-lesioned group, whereas the percentages were only around $10 \%$ in the 6-OHDAlesioned group (control vs PD, $\chi^{2}=46.83, p<0.001$; L-DOPA-off vs dyskinesia-off, $\chi^{2}=45.37, p<0.001$; L-DOPA-on vs dyskinesia-on, $\chi^{2}=32.98, p<0.001 ; \chi^{2}$ test with Bonferroni's correction; Table 1). There were no significant differences in the number of peaks of autocorrelograms and the mean time of the first peak (Table 1).

Population PSDs of SNr neurons revealed that 50- to $100-\mathrm{Hz}$ activity was high, while $10-$ to $50-\mathrm{Hz}$ activity was low in all three states in the sham-lesioned group (Fig. $4 B$, left). SNr neurons in all three states in the 6-OHDA-lesioned group exhibited low-frequency oscillation around $1 \mathrm{~Hz}$ (Fig. $4 B$, right, *).

\section{Changes of $\mathrm{Cx}$-evoked responses of GPe neurons}

Next, we examined the responses of GPe neurons induced by $\mathrm{Cx}$ stimulation by constructing PSTHs (Fig. 5A). The typical response pattern of GPe neurons in the control state of the sham-lesioned group was a triphasic response composed of early excitation, inhibition, and late excitation (Fig. 5B, control), as reported previously (Chiken et al., 2008, 2015; Sano et al., 2013;
Sano and Nambu, 2019). Each component in the GPe is mediated by the Cx-STN-GPe, Cx-Stri $-\mathrm{GPe}$, and Cx-Stri-GPe-STNGPe pathways, respectively (Fig. 5A; Ryan and Clark, 1991; Nambu et al., 2000; Kita et al., 2004; Sano et al., 2013). This typical response pattern was not changed in the L-DOPA-off and LDOPA-on states (Fig. 5B, L-DOPA-off, L-DOPA-on). These findings were also confirmed in population PSTHs (Fig. 5C, left), bar graphs categorizing response patterns (Fig. $5 D$, upper), heat map visualization (Fig. $5 E$, upper), and quantitative analyses (GPe, sham lesioned; Table 2).

Similar to the sham-lesioned group, the most common response pattern in the $\mathrm{PD}$, dyskinesia-off, and dyskinesia-on states of the 6-OHDA-lesioned group was a triphasic response (Fig. $5 B, \mathrm{PD}$, dyskinesia-off, dyskinesia-on, $D$, lower). However, late excitation was strengthened in the PD and dyskinesia-off states, and weakened in the dyskinesia-on state (Fig. 5B, PD, dyskinesia-off, dyskinesia-on). Population PSTHs (Fig. 5C, right) and heat maps (Fig. 5E, lower) depict these differences in late excitation.

Quantitative analyses confirmed these changes (GPe, 6OHDA lesioned; Table 2). Both the duration and amplitude of 
Table 2. Response parameters of GPe and SNr neurons to $\mathrm{Cx}$ stimulation

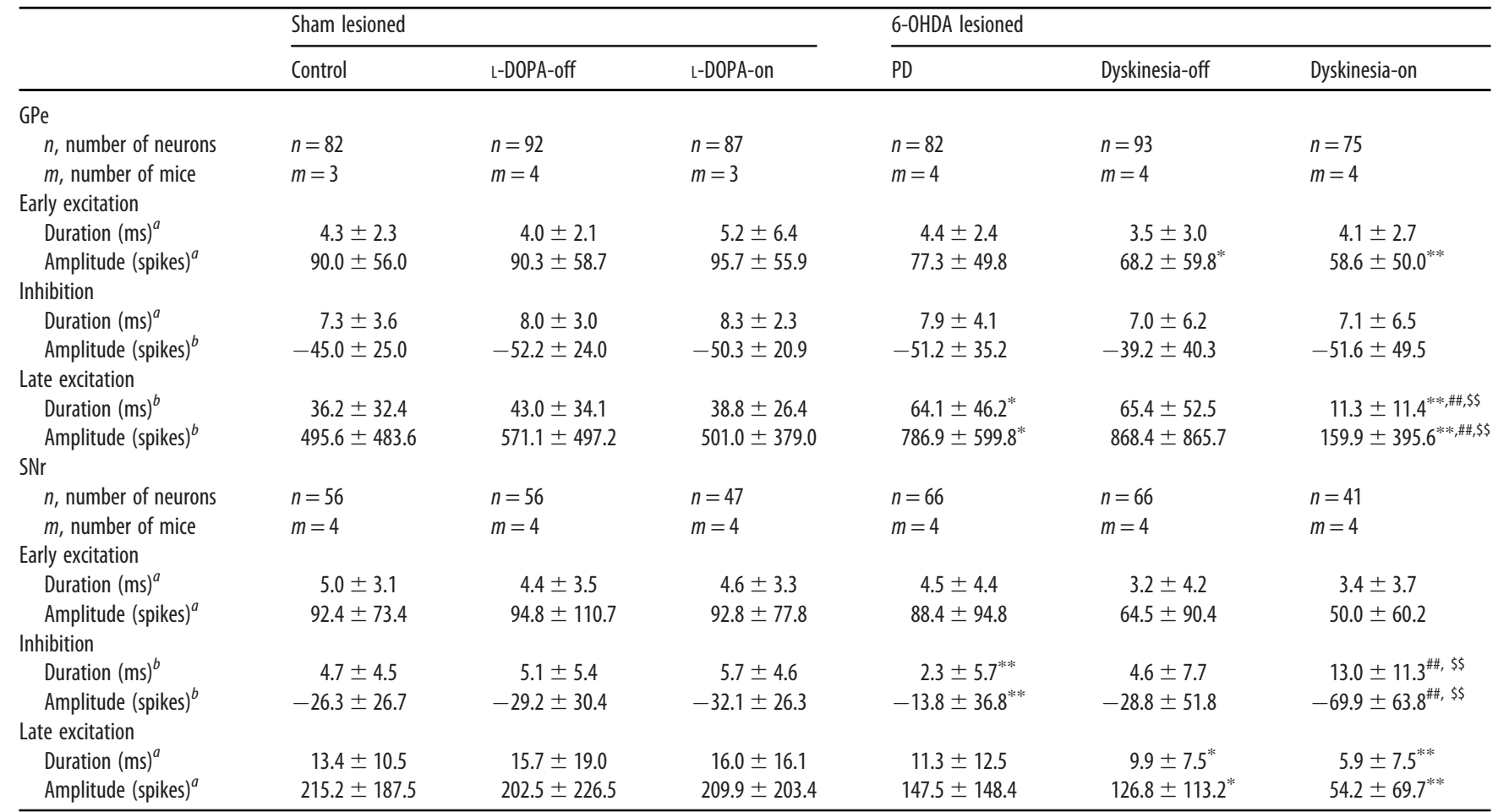

Values are means $\pm S D, * p<0.05, * * p<0.01$ significantly different from the corresponding states of the sham-lesioned group, \#\#p $<0.01$ significantly different from the PD state, $\$ \$ p<0.01$ significantly different from the dyskinesia-off state; ${ }^{a}$ two-way ANOVA (no interaction between lesion group $\times$ L-DOPA treatment) followed by Bonferroni's post hoc test, ${ }^{b}$ two-way ANOVA (significant interaction between lesion group $\times$ L-DOPA treatment) followed by Kruskal-Wallis test with Dunn's multiple comparison test.

late excitation (duration, significant interaction between lesion group $\times$ L-DOPA treatment, $F_{(2,505)}=29.04, p<0.001$; amplitude, $F_{(2,505)}=17.6, p<0.001$; two-way ANOVA) were significantly larger in the PD state and smaller in the dyskinesia-on state (duration, control vs PD, $p=0.011$; L-DOPA-on vs dyskinesia-on, $p<0.001$; PD vs dyskinesia-on, $p<0.001$; dyskinesia-off vs dyskinesia-on, $p<0.001$; Kruskal-Wallis test with Dunn's multiple comparison test; amplitude, control vs PD, $p=0.01$; LDOPA-on vs dyskinesia-on, $p=0.001$; PD vs dyskinesia-on, $p<0.001$; dyskinesia-off vs dyskinesia-on, $p<0.001)$. The amplitude of early excitation (significant effect of lesion group, $F_{(1,505)}=23.77, p=0.01$; two-way ANOVA) was smaller in the dyskinesia-off and dyskinesia-on states in the 6-OHDA-lesioned group as compared with the sham-lesioned group (L-DOPA-off vs dyskinesia-off, $p=0.021$; L-DOPA-on vs dyskinesia-on, $p<0.001$; Bonferroni's post hoc test).

\section{Changes of $\mathrm{Cx}$-evoked responses of $\mathrm{SNr}$ neurons}

We also examined $\mathrm{Cx}$-evoked responses of $\mathrm{SNr}$ neurons (Fig. $6 A)$. The typical response pattern of $\mathrm{SNr}$ neurons in the control state of the sham-lesioned group was a triphasic response composed of early excitation, inhibition, and late excitation (Fig. 6B, control), as reported previously (Sano et al., 2013; Sano and Nambu, 2019). Each component in the SNr/GPi is mediated by the Cx-STN-SNr/GPi hyperdirect, Cx-Str ${ }_{\mathrm{d}}-\mathrm{SNr} / \mathrm{GPi}$ direct, and $\mathrm{Cx}-\mathrm{Str}_{\mathrm{i}}-\mathrm{GPe}-\mathrm{STN}-\mathrm{SNr} / \mathrm{GPi}$ indirect pathways, respectively (Fig. 6A; Maurice et al., 1999; Nambu et al., 2000; Tachibana et al., 2008; Sano et al., 2013). This typical response pattern was not changed in the L-DOPA-off and L-DOPA-on states (Fig. 6B, LDOPA-off, L-DOPA-on). These findings were also confirmed in population PSTHs (Fig. 6C, left), bar graphs (Fig. 6D, upper), heat maps (Fig. $6 E$, upper), and quantitative analyses (SNr, sham lesioned; Table 2).
On the other hand, in the PD state of the 6-OHDA-lesioned group, monophasic excitation was the typical response pattern (Fig. 6B, PD). Population PSTHs also showed monophasic excitation (Fig. 6C, right, PD). Excitation without inhibition, such as monophasic early excitation, biphasic excitation, and monophasic late excitation, became dominant (80\%; Fig. 6D, PD, warm colors), and responses with inhibition, such as a triphasic response, inhibition followed by excitation, excitation followed by inhibition, and monophasic inhibition, was reduced in the PD state $\left(20 \% ; \chi^{2}=51.24, p<0.001, \chi^{2}\right.$ test with Bonferroni's correction; Fig. $6 D, \mathrm{PD}$, cold colors). In the heat map, it is apparent that monophasic excitation was dominant, and a fraction of biphasic responses composed of early excitation and the following inhibition was observed (Fig. 6E, PD).

After chronic L-DOPA treatment, in the dyskinesia-off state, excitation without inhibition (Fig. $6 B$, dyskinesia-off, $C$, right, dyskinesia-off) remained dominant (65\%; Fig. $6 D$, dyskinesiaoff, warm colors). However, responses with inhibition and without any late excitation, such as excitation followed by inhibition and monophasic inhibition (15\%; Fig. 6D, dyskinesia-off, greenish colors), were increased in number compared with the PD state $\left(3 \% ; \chi^{2}=6.54, p=0.015\right)$. These changes were observed as a small deflection in the falling phase of population PSTHs (Fig. $6 C$, right, dyskinesia-off, red arrowhead). In the heat map, inhibition was increased, although monophasic excitation remained (Fig. 6E, dyskinesia-off).

In the dyskinesia-on state, the typical response pattern was a biphasic response composed of excitation followed by inhibition (Fig. 6B, dyskinesia-on). Responses with inhibition and without late excitation (44\%; Fig. 6D, dyskinesia-on, greenish colors) were increased in number compared with $\mathrm{PD}$ and dyskinesia-off states $\left(\chi^{2}=34.98, p<0.001\right)$. Meanwhile, responses without inhibition (32\%; Fig. $6 D$, dyskinesia-on, warm colors) were 

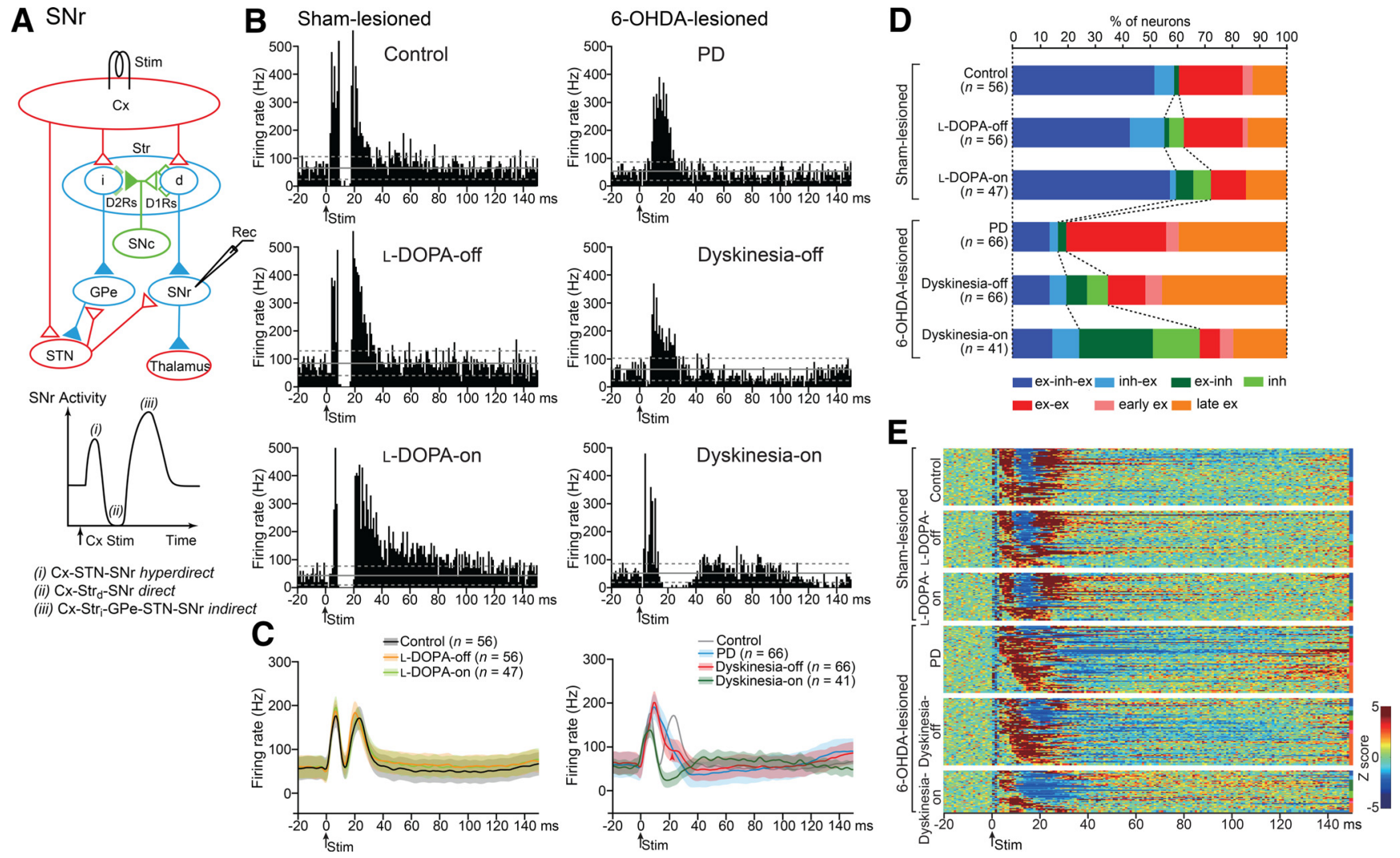

Figure 6. Responses of $S N r$ neurons evoked by motor $\mathrm{Cx}$ stimulation. $\boldsymbol{A}$, The stimulation $(\mathrm{Cx})$ and recording $(\mathrm{SNr})$ sites are depicted with the $\mathrm{BG}$ circuitry. $\mathrm{Cx}$-evoked response in the $\mathrm{SNr}$ is typically composed of early excitation (i), inhibition (ii), and following late excitation (iii), which are mediated by the CX-STN-SNr hyperdirect, Cx-Str ${ }_{d}$-SNr direct, and CX-Stri-GPe-STN-SNr indirect pathways, respectively. B, PSTHs of the typical response of SNr neurons to Cx stimulation. C, Population PSTHs of Cx-evoked responses in SNr neurons. D, Response patterns of SNr neurons. $\boldsymbol{E}$, Heat maps of CX-evoked responses of SNr neurons.

reduced in the dyskinesia-on state compared with the PD and dyskinesia-off states $\left(p<0.001, \chi^{2}=34.98\right)$. Population PSTHs revealed that the averaged response in the dyskinesia-on state was excitation followed by inhibition without late excitation (Fig. $6 \mathrm{C}$, right, dyskinesia-on). In the heat map, inhibition appeared to be further increased, and late excitation was mostly lost (Fig. 6E, dyskinesia-on).

Quantitative analysis ( $\mathrm{SNr}, 6-\mathrm{OHDA}$ lesioned; Table 2) revealed that the duration and amplitude of inhibition (duration, significant interaction between lesion group $\times \mathrm{L}$-DOPA treatment, $F_{(2,326)}=15.45, p<0.001$; amplitude, $F_{(2,326)}=11.07, p<0.001$; two-way ANOVA) were significantly smaller in the PD state (duration, $p=0.005$; amplitude, $p=0.004$; Kruskal-Wallis test with Dunn's multiple comparison test) and returned to the control levels in the dyskinesia-off state. The duration and amplitude of inhibition were further increased in the dyskinesia-on state (duration, PD vs dyskinesia-on, $p<0.001$; dyskinesia-off vs dyskinesia-on, $p<0.001$; amplitude, PD vs dyskinesia-on, $p<0.001$; dyskinesiaoff vs dyskinesia-on, $p<0.001$ ), reflecting the dominance of inhibition in the dyskinesia-on state. The duration and amplitude of late excitation (duration, significant effect of lesion group, $F_{(1,326)}=17.62, p<0.001$; amplitude, $F_{(1,326)}=28.67, p<0.001$; two-way ANOVA) was significantly smaller in the dyskinesia-off (duration, $p=0.038$; amplitude, $p=0.039$; Bonferroni's post hoc test) and dyskinesia-on (duration, $p<0.001$; amplitude, $p<0.001$ ) states, as compared with the sham-lesioned group.

\section{Location of recorded GPe and SNr neurons}

Recording sites were plotted in the representative frontal planes of the GPe and $\mathrm{SNr}$ with different symbols based on Cx-evoked response patterns (Fig. 7). In the GPe, we mainly recorded in the middle and lateral parts in both the sham-lesioned and 6OHDA-lesioned groups (Fig. 7A), which corresponds to the somatomotor region of the GPe (Chiken et al., 2008, 2015; Sano et al., 2013). In the control, L-DOPA-off, and L-DOPA-on states of the sham-lesioned group, the most common response pattern was a triphasic response composed of early excitation, inhibition, and late excitation (Fig. 7A, left, blue circles). This common response pattern remained unchanged in the $\mathrm{PD}$, dyskinesia-off, and dyskinesia-on states of the 6-OHDA-lesioned group, with similar distribution in the GPe (Fig. 7A, right, blue circles).

In the $\mathrm{SNr}$, we mainly recorded in the dorso-lateral part in both the sham-lesioned and 6-OHDA-lesioned groups (Fig. 7B), which corresponds to the somatomotor region of the $\mathrm{SNr}$ (Sano et al., 2013). In the sham-lesioned group, the most common response pattern was a triphasic response (Fig. $7 B$, left, blue circles). In the PD state, the most common response pattern was excitation without inhibition (Fig. $7 B$, right, $\mathrm{PD}$, warm colors) and remained unchanged in the dyskinesia-off state (Fig. $7 B$, right, dyskinesia-off). In the dyskinesia-on state, the most common response pattern was inhibition without late excitation, such as excitation-inhibition and monophasic inhibition (Fig. 7B, right, dyskinesia-on, greenish colors). $\mathrm{SNr}$ neurons with these response patterns were similarly distributed to those in the sham-lesioned group, suggesting that $\mathrm{SNr}$ neurons in the same area showed different response patterns between the control, PD, dyskinesia-off, and dyskinesia-on states.

\section{Discussion}

To elucidate the pathophysiology of LID, we recorded neuronal activity in the GPe and SNr of model mice. (1) There were 

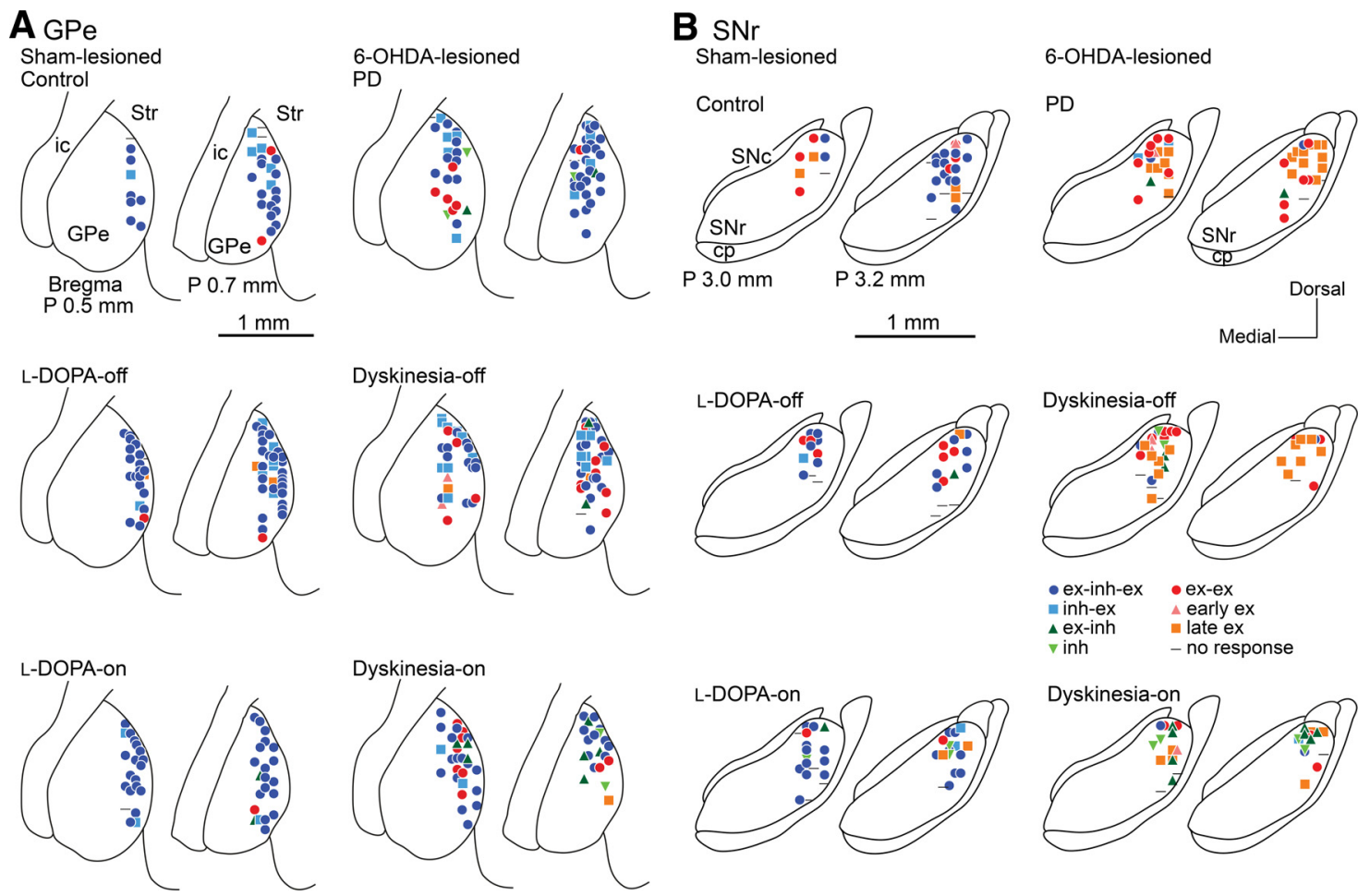

Figure 7. Distribution of recorded GPe and SNr neurons. $\boldsymbol{A}, \boldsymbol{B}$, Neurons recorded in representative frontal sections in the GPe $(\boldsymbol{A}$, posterior 0.5 and $0.7 \mathrm{~mm}$ from bregma) and SNr $(\boldsymbol{B}$, posterior 3.0 and $3.2 \mathrm{~mm}$ from bregma) of all mice in the control, L-DOPA-off, and L-DOPA-on states of the sham-lesioned group (left), and in the PD, dyskinesia-off, and dyskinesia-on states of the 6-OHDA-lesioned group (right) are indicated by different symbols based on their response patterns. cp, cerebral peduncle; ic, internal capsule.

almost no differences in the spontaneous firing rates and patterns, and $\mathrm{Cx}$-evoked responses of $\mathrm{GPe}$ and $\mathrm{SNr}$ neurons between the control, L-DOPA-off, and L-DOPA-on states. (2) Spontaneous firing rates of GPe neurons were decreased in the dyskinesia-off state and increased in the dyskinesia-on state, while those of SNr neurons showed no changes. (3) GPe and SNr neurons generally increased bursting activity and low-frequency oscillation around $1 \mathrm{~Hz}$ in the $\mathrm{PD}$, dyskinesia-off, and dyskinesia states. (4) In the GPe, Cx-evoked late excitation was increased in the PD and dyskinesia-off states, but decreased in the dyskinesiaon state. (5) In the $\mathrm{SNr}, \mathrm{Cx}$-evoked inhibition was largely suppressed in the PD state, but enhanced in the dyskinesia-on state, and $\mathrm{Cx}$-evoked late excitation was suppressed in the dyskinesiaoff and dyskinesia-on states, indicating specific changes to LID.

\section{Spontaneous activity changes in the GPe and $\mathrm{SNr}$}

Dopaminergic inputs inhibit $\operatorname{Str}_{\mathrm{i}}$ neurons through dopamine D2 receptors (D2Rs) and stimulate $\operatorname{Str}_{\mathrm{d}}$ neurons through dopamine D1 receptors (D1Rs; Figs. 5A, 6A; Albin et al., 1989; DeLong, 1990; Gerfen et al., 1990). Thus, loss of dopamine in PD would increase the activity of $\operatorname{Str}_{i}$ neurons and conversely decrease the activity of $\mathrm{Str}_{\mathrm{d}}$ neurons, resulting in hypoactivity of GPe neurons and hyperactivity of SNr/GPi neurons. On the other hand, excessive dopamine input would induce hyperactivity of GPe neurons and hypoactivity of SNr/GPi neurons, resulting in LID (Crossman, 1990; Bezard et al., 2001) in PD patients (Hutchinson et al., 1997; Lozano et al., 2000) and monkeys (Filion et al., 1991; Papa et al., 1999; Boraud et al., 2001). However, recent studies in PD do not support these spontaneous activity changes (Wichmann et al., 1999; Wichmann and Soares, 2006; Galvan et al., 2010; Tachibana et al., 2011). The present study also showed no firing rate changes of SNr neurons in the PD, dyskinesia-off, and dyskinesia-on states (Fig. 2).
Bursts and oscillatory activity in the BG were reported to be correlated with PD motor features (Wichmann et al., 1999; Heimer et al., 2002; Wichmann and Soares, 2006; Tachibana et al., 2011). Low-frequency $(0.5-4 \mathrm{~Hz})$ oscillatory activity was also reported in dopamine-depleted states (Walters et al., 2007; Aristieta et al., 2016; Whalen et al., 2020). Low-frequency oscillations were found in the STN of dyskinetic PD patients (Foffani et al., 2005; Alonso-Frech et al., 2006) and in the SNr of a rat LID model (Meissner et al., 2006). In the present study, we found firing pattern changes (Figs. 3,4), but they were not specific to LID.

\section{Changes of $\mathrm{Cx}$-evoked responses in the $\mathrm{GPe}$}

In the GPe, $\mathrm{Cx}$ stimulation induces early excitation, inhibition, and late excitation, which is mediated by the Cx-STN-GPe, Cx$\mathrm{Str}_{\mathrm{i}}-\mathrm{GPe}$, and $\mathrm{Cx}-\mathrm{Str}_{\mathrm{i}}-\mathrm{GPe}-\mathrm{STN}-\mathrm{GPe}$ pathways, respectively (Figs. 5A, 8, GPe, normal). In the present study, 6-OHDA lesion strengthened $\mathrm{Cx}$-evoked late excitation in GPe neurons, which persisted after chronic L-DOPA treatment in the dyskinesia-off state, but was lessened in the dyskinesia-on state (Fig. 5; Table 2), suggesting that neurotransmission through the $\mathrm{Cx}-\mathrm{Str}_{\mathrm{i}}-\mathrm{GPe}-$ STN-GPe pathway is upregulated in the PD and dyskinesia-off states, and downregulated in the dyskinesia-on state (Fig. 8, $\mathrm{GPe}$ ). Similar changes were reported in rodent PD models (Kita and Kita, 2011; Sano and Nambu, 2019). A plausible mechanism is alteration in the Cx-Str ${ }_{i}$ neurotransmission; following loss of inhibitory dopamine signals through D2Rs, Str $_{i}$ neurons increased their intrinsic excitability (Suárez et al., 2016; Parker et al., 2018; Ryan et al., 2018), and in the dyskinesia-on state, decreased their activity (Parker et al., 2018; Ryan et al., 2018) or returned to the control excitability (Suárez et al., 2016).

However, in the present study, Cx-evoked inhibition in the GPe mediated by the Cx-Str ${ }^{-}$-GPe pathway did not show evident 


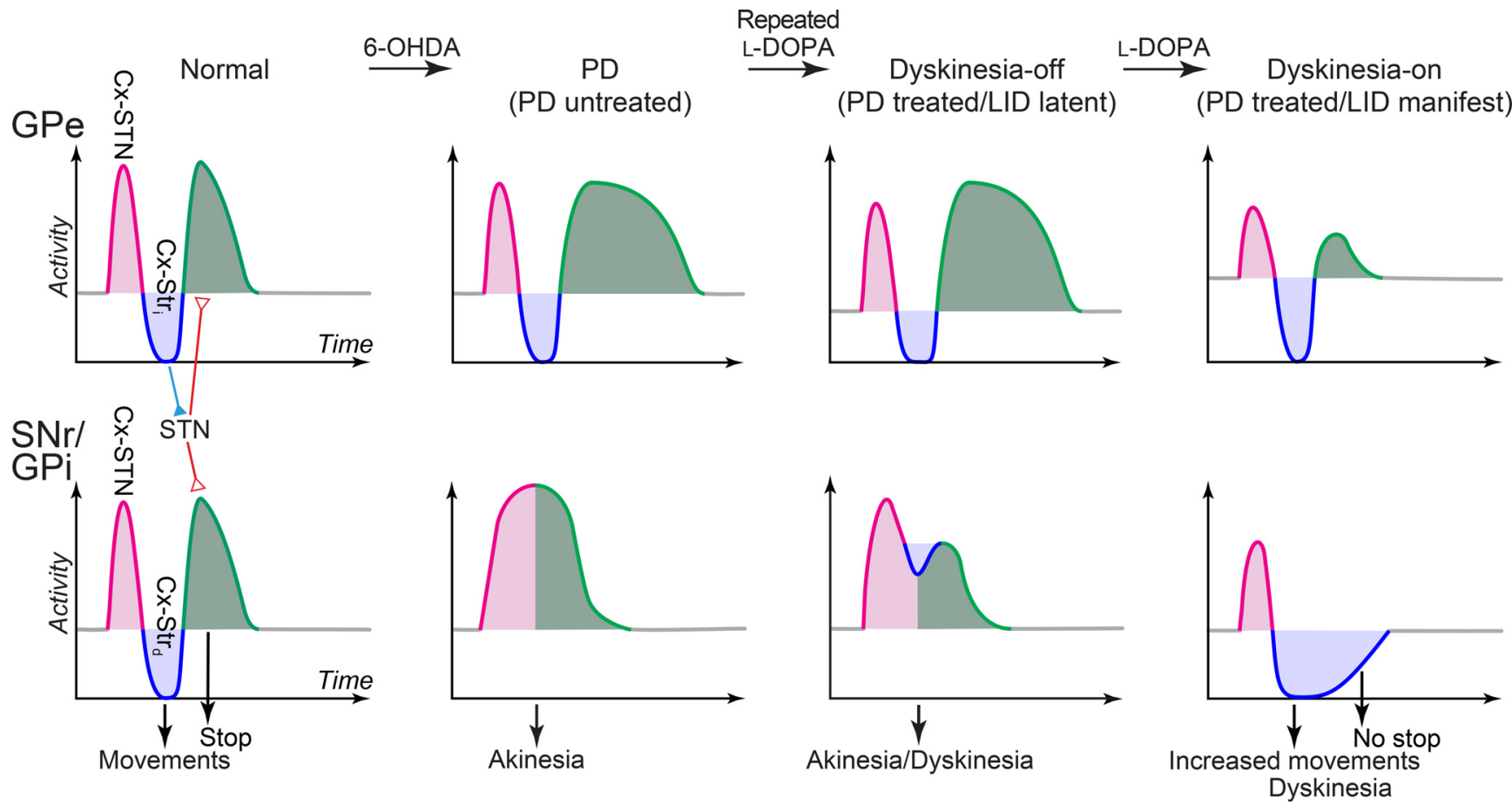

Figure 8. Schematic diagram of (x-evoked responses in the GPe (upper) and SNr/GPi (lower) and the pathophysiology of PD and LID based on our dynamic model of the BG circuitry. In the normal state, $C x$-induced excitation (magenta) in the SNr/GPi through the CX-STN-SNr/GPi hyperdirect pathway resets on-going activity in the thalamus and Cx, the following Cx-induced inhibition (blue) in the SNr/GPi through the $\mathrm{Cx}_{-} \mathrm{Str}_{\mathrm{d}}-\mathrm{SNr} / \mathrm{GPi}$ direct pathway disinhibits the thalamus/Cx and releases intended movements at an appropriate timing, and then, $\mathrm{C}$-induced late excitation in the SNr/GPi (green) through the (x-Stri-GPe-STN-SNr/GPi indirect pathway stops movements. In the PD state (PD untreated), reduced inhibition in the SNr/GPi cannot release movements, resulting in akinesia. After repeated L-DOPA treatment, in the dyskinesia-off state (PD treated/LID latent), recovered inhibition (blue) and reduced late excitation (green) in the SNr/GPi partially restores movements, reflecting latent changes in LID. In the dyskinesia-on state (PD treated/LID manifest), L-DOPA application further enhances inhibition (blue) and suppresses late excitation in the SNr/GPi, leading to excessively increased movements that cannot be stopped, manifesting as dyskinesia.

changes in the PD and dyskinesia-off states (Fig. 5; Table 2), probably because (1) Str $r_{i}$ neurons, which were fully activated in the control state, could not further inhibit GPe activity, and/or (2) increased late excitation may mask increased inhibition. Decreased early excitation in the dyskinesia-off state (Table 2) may reflect increased inhibition. Another plausible mechanism is that dopamine depletion strengthens GPe-STN neurotransmission directly (Fan et al., 2012) or indirectly through the Cx-STN projections (Chu et al., 2015, 2017).

\section{Changes of $\mathrm{Cx}$ evoked responses in the $\mathrm{SNr}$}

In the $\mathrm{SNr}, \mathrm{Cx}$ stimulation induces early excitation, inhibition, and late excitation, which is mediated by the Cx-STN-SNr hyperdirect, $\mathrm{Cx}-\mathrm{Str}_{\mathrm{d}}-\mathrm{SNr}$ direct, and $\mathrm{Cx}-\mathrm{Str}_{\mathrm{i}}-\mathrm{GPe}-\mathrm{STN}-\mathrm{SNr}$ indirect pathways, respectively (Figs. 6A, 8, SNr/GPi, normal). Cx-evoked inhibition in $\mathrm{SNr}$ neurons was lost in the PD state, partially recovered in the dyskinesia-off state, and strongly enhanced in the dyskinesia-on state (Fig. 6; Table 2), suggesting that inhibitory inputs through the $\mathrm{Cx}-\mathrm{Str}_{\mathrm{d}} \mathrm{-SNr}$ direct pathway are downregulated in the PD state, partially recovered in the dyskinesia-off state, and upregulated in the dyskinesia-on state (Fig. 8, $\mathrm{SNr} /$ GPi). Similar changes were reported in PD rodents and in D1Rs knockdown mice (Kita and Kita, 2011; Chiken et al., 2015; Sano and Nambu, 2019). These changes could be attributed to activity changes of $\operatorname{Str}_{\mathrm{d}}$ neurons. Dopamine depletion reduced the activity of $\operatorname{Str}_{d}$ neurons (Mallet et al., 2006; Parker et al., 2018; Ryan et al., 2018) and decreased their spine densities (Suárez et al., 2014, 2016). In the dyskinesia-off state, the activity of $\operatorname{Str}_{\mathrm{d}}$ neurons was increased (Fieblinger et al., 2014; Suárez et al., 2016), and in the dyskinesia-on state, was further increased (Parker et al., 2018;
Ryan et al., 2018). Str $_{d}$ activity has a causative role in LID; optogenetic activation of $\operatorname{Str}_{\mathrm{d}}$ neurons or both $\operatorname{Str}_{\mathrm{d}}$ and $\mathrm{Str}_{\mathrm{i}}$ neurons (Hernández et al., 2017; Perez et al., 2017; Girasole et al., 2018; Keifman et al., 2019) or activation of D1Rs (Darmopil et al., 2009) induced LID. Increased GABA release from the $\operatorname{Str}_{d}-\mathrm{SNr}$ terminals may also contribute to the increased $\mathrm{Cx}$-induced inhibition (Yamamoto et al., 2006; Borgkvist et al., 2015).

In the PD state, early excitation mediated by the Cx-STN-SNr hyperdirect pathway and late excitation mediated by the $\mathrm{Cx}-\mathrm{Str}_{\mathrm{i}^{-}}$ GPe-STN-SNr indirect pathway fused together in the SNr, forming monophasic excitation (Fig. 8, $\mathrm{SNr} / \mathrm{GPi}, \mathrm{PD}$ ). In the dyskinesia-off state, late excitation in $\mathrm{SNr}$ neurons was reduced (Fig. 6; Table 2), probably because increased late excitation in the GPe could depress late excitation in $\mathrm{SNr}$ neurons through the inhibitory GPe-SNr and/or GPe-STN-SNr pathways (Fig. 8, SNr/GPi, dyskinesia-off). In the dyskinesia-on state, $\mathrm{Cx}$-evoked late excitation in $\mathrm{SNr}$ neurons was further depressed (Fig. 6; Table 2), which could be explained by the downregulation of the $\mathrm{Cx}-\mathrm{Str}_{\mathrm{i}}{ }^{-}$ GPe-STN pathway as discussed above (Fig. 8, SNr/GPi, dyskinesia-on).

In the present study, each connection of $\mathrm{Cx}$-BG pathways was not directly evaluated. Further studies using pathway specific manipulation are necessary to clarify the origin of changes in Cx-evoked responses.

\section{Pathophysiology of PD and LID}

We have proposed a dynamic model of the Cx-BG network (Nambu et al., 2000, 2002, 2015) to explain the control mechanism of voluntary movements (Fig. 8, normal). First, $\mathrm{Cx}$-induced early excitation (magenta) in the $\mathrm{SNr} / \mathrm{GPi}$ through the $\mathrm{Cx}-\mathrm{STN}$ - 
SNr/GPi hyperdirect pathway resets on-going activity in the thalamus and Cx. Second, inhibition (blue) in the SNr/GPi through the $\mathrm{Cx}-\mathrm{Str}_{\mathrm{d}}-\mathrm{SNr} / \mathrm{GPi}$ direct pathway disinhibits thalamus/Cx, and releases intended movements at an appropriate timing. Finally, late excitation (green) in the SNr/GPi through the CxStr $_{\mathrm{i}}-\mathrm{GPe}-\mathrm{STN}-\mathrm{SNr} / \mathrm{GPi}$ indirect pathway inhibits thalamus/Cx and stops movements. Based on this model and the present study, we can explain the pathophysiology of PD and LID (Fig. 8).

(1) In the PD state (PD untreated), inhibition in the SNr/GPi mediated by the direct pathway is suppressed, and early excitation mediated by the hyperdirect pathway and late excitation mediated by the indirect pathway become dominant. Thus, signals through the direct pathway cannot release an appropriate movement at an appropriate timing, resulting in akinesia.

(2) In the dyskinesia-off state (PD treated/LID latent), inhibition through the direct pathway is partially recovered, and late excitation through the indirect pathway is suppressed in the $\mathrm{SNr}$ / GPi. This may be a latent change in LID. In the present study, some PD motor features, such as abnormal rotational behaviors, were reduced in the dyskinesia-off state.

(3) In the dyskinesia-on state (PD treated/LID manifest), L-DOPA application further enhances inhibition and depresses late excitation in the $\mathrm{SNr} / \mathrm{GPi}$. This situation means that signals to release movements through the direct pathway are enhanced, while signals to stop movements through the indirect pathway are suppressed. Thus, unintended movements can be easily released at random timing and cannot be easily stopped once they are released, which is the manifestation of dyskinesia.

\section{Clinical significance}

LID is one of the major side effects after long-term L-DOPA treatment, and its control is a major issue for advanced PD. Increased inhibition mediated by the direct pathway and loss of late excitation mediated by the indirect pathway in the SNr/GPi seem to play a causative role in LID (Fig. 8). Restoring neurotransmission through the indirect pathway and/or suppressing neurotransmission through the direct pathway (Darmopil et al., 2009) may be useful to suppress LID, suggesting a future therapeutic strategy for LID.

\section{References}

Ahlskog JE, Muenter MD (2001) Frequency of levodopa-related dyskinesias and motor fluctuations as estimated from the cumulative literature. Mov Disord 16:448-458.

Albin RL, Young AB, Penney JB (1989) The functional anatomy of basal ganglia disorders. Trends Neurosci 12:366-375.

Alcacer C, Andreoli L, Sebastianutto I, Jakobsson J, Fieblinger T, Cenci MA (2017) Chemogenetic stimulation of striatal projection neurons modulates responses to Parkinson's disease therapy. J Clin Invest 127:720-734.

Alexander GE, Crutcher MD (1990) Functional architecture of basal ganglia circuits: neural substrates of parallel processing. Trends Neurosci 13:266271.

Alonso-Frech F, Zamarbide I, Alegre M, Rodríguez-Oroz MC, Guridi J, Manrique M, Valencia M, Artieda J, Obeso JA (2006) Slow oscillatory activity and levodopa-induced dyskinesias in Parkinson's disease. Brain J Neurol 129:1748-1757.

Aristieta A, Ruiz-Ortega JA, Miguelez C, Morera-Herreras T, Ugedo L (2016) Chronic L-DOPA administration increases the firing rate but does not reverse enhanced slow frequency oscillatory activity and synchronization in substantia nigra pars reticulata neurons from 6-hydroxydopaminelesioned rats. Neurobiol Dis 89:88-100.
Bezard E, Brotchie JM, Gross CE (2001) Pathophysiology of levodopainduced dyskinesia: potential for new therapies. Nat Rev Neurosci 2:577588.

Boraud T, Bezard E, Guehl D, Bioulac B, Gross C (1998) Effects of L-DOPA on neuronal activity of the globus pallidus externalis (GPe) and globus pallidus internalis (GPi) in the MPTP-treated monkey. Brain Res 787:157-160.

Boraud T, Bezard E, Bioulac B, Gross CE (2001) Dopamine agonist-induced dyskinesias are correlated to both firing pattern and frequency alterations of pallidal neurones in the MPTP-treated monkey. Brain J Neurol 124:546-557.

Borgkvist A, Avegno EM, Wong MY, Kheirbek MA, Sonders MS, Hen R, Sulzer D (2015) Loss of striatonigral GABAergic presynaptic inhibition enables motor sensitization in parkinsonian mice. Neuron 87: 976-988.

Cenci MA (2007) Dopamine dysregulation of movement control in LDOPA-induced dyskinesia. Trends Neurosci 30:236-243.

Cenci MA (2014) Presynaptic mechanisms of 1-DOPA-induced dyskinesia: the findings, the debate, and the therapeutic implications. Front Neurol $5: 242$.

Cenci MA, Lundblad M (2006) Post- versus presynaptic plasticity in LDOPA-induced dyskinesia. J Neurochem 99:381-392.

Cenci MA, Lundblad M (2007) Ratings of L-DOPA-induced dyskinesia in the unilateral 6-OHDA lesion model of Parkinson's disease in rats and mice. Curr Protoc Neurosci Chapter 9:Unit 9.25.

Chiken S, Shashidharan P, Nambu A (2008) Cortically evoked long-lasting inhibition of pallidal neurons in a transgenic mouse model of dystonia. J Neurosci 28:13967-13977.

Chiken S, Sato A, Ohta C, Kurokawa M, Arai S, Maeshima J, SunayamaMorita T, Sasaoka T, Nambu A (2015) Dopamine D1 receptor-mediated transmission maintains information flow through the cortico-striatoentopeduncular direct pathway to release movements. Cereb Cortex 25:4885-4897.

Chu HY, Atherton JF, Wokosin D, Surmeier DJ, Bevan MD (2015) Heterosynaptic regulation of external globus pallidus inputs to the subthalamic nucleus by the motor cortex. Neuron 85:364-376.

Chu HY, McIver EL, Kovaleski RF, Atherton JF, Bevan MD (2017) Loss of hyperdirect pathway cortico-subthalamic inputs following degeneration of midbrain dopamine neurons. Neuron 95:1306-1318.e5.

Cotzias GC, Papavasiliou PS, Gellene R (1969) Modification of parkinsonism - chronic treatment with L-dopa. N Engl J Med 280:337-345.

Crossman AR (1990) A hypothesis on the pathophysiological mechanisms that underlie levodopa- or dopamine agonist-induced dyskinesia in Parkinson's disease: implications for future strategies in treatment. Mov Disord 5:100-108.

Darmopil S, Martín AB, De Diego IR, Ares S, Moratalla R (2009) Genetic inactivation of dopamine D1 but not D2 receptors inhibits L-DOPAinduced dyskinesia and histone activation. Biol Psychiatry 66:603-613.

DeLong MR (1990) Primate models of movement disorders of basal ganglia origin. Trends Neurosci 13:281-285.

Fahn S (2000) The spectrum of levodopa-induced dyskinesias. Ann Neurol 47 [4 Suppl 1]:S2-S9; discussion S9-S11.

Fahn S (2003) Description of Parkinson's disease as a clinical syndrome. Ann NY Acad Sci 991:1-14.

Fan KY, Baufreton J, Surmeier DJ, Chan CS, Bevan MD (2012) Proliferation of external globus pallidus-subthalamic nucleus synapses following degeneration of midbrain dopamine neurons. J Neurosci 32:1371813728.

Fieblinger T, Graves SM, Sebel LE, Alcacer C, Plotkin JL, Gertler TS, Chan CS, Heiman M, Greengard P, Cenci MA, Surmeier DJ (2014) Cell typespecific plasticity of striatal projection neurons in parkinsonism and LDOPA-induced dyskinesia. Nat Commun 5:5316.

Filion M, Tremblay L (1991) Abnormal spontaneous activity of globus pallidus neurons in monkeys with MPTP-induced parkinsonism. Brain Res 547:140-144.

Filion M, Tremblay L, Bédard PJ (1991) Effects of dopamine agonists on the spontaneous activity of globus pallidus neurons in monkeys with MPTPinduced parkinsonism. Brain Res 547:145-149.

Foffani G, Bianchi A, Baselli G, Priori A (2005) Movement-related frequency modulation of beta oscillatory activity in the human subthalamic nucleus. J Physiol 568:699-711. 
Francardo V, Recchia A, Popovic N, Andersson D, Nissbrandt H, Cenci MA (2011) Impact of the lesion procedure on the profiles of motor impairment and molecular responsiveness to L-DOPA in the 6-hydroxydopamine mouse model of Parkinson's disease. Neurobiol Dis 42:327-340.

Franklin KBJ, Paxinos G (2008) The mouse brain in stereotaxic coordinates. San Diego: Academic Press.

Galvan A, Hu X, Smith Y, Wichmann T (2010) Localization and function of GABA transporters in the globus pallidus of parkinsonian monkeys. Exp Neurol 223:505-515.

Gerfen CR, Engber TM, Mahan LC, Susel Z, Chase TN, Monsma FJ, Sibley DR (1990) D1 and D2 dopamine receptor-regulated gene expression of striatonigral and striatopallidal neurons. Science 250:1429-1432.

Girasole AE, Lum MY, Nathaniel D, Bair-Marshall CJ, Guenthner CJ, Luo L, Kreitzer AC, Nelson AB (2018) A subpopulation of striatal neurons mediates levodopa-induced dyskinesia. Neuron 97:787-795.e6.

Heimer G, Bar-Gad I, Goldberg JA, Bergman H (2002) Dopamine replacement therapy reverses abnormal synchronization of pallidal neurons in the 1-methyl-4-phenyl-1,2,3,6-tetrahydropyridine primate model of parkinsonism. J Neurosci 22:7850-7855.

Heimer G, Rivlin-Etzion M, Bar-Gad I, Goldberg JA, Haber SN, Bergman H (2006) Dopamine replacement therapy does not restore the full spectrum of normal pallidal activity in the 1-methyl-4-phenyl-1,2,3,6-tetra-hydropyridine primate model of parkinsonism. J Neurosci 26:8101-8114.

Hernández LF, Castela I, Ruiz -DeDiego I, Obeso JA, Moratalla R (2017) Striatal activation by optogenetics induces dyskinesias in the 6-hydroxydopamine rat model of Parkinson disease. Mov Disord 32:530-537.

Hutchinson WD, Levy R, Dostrovsky JO, Lozano AM, Lang AE (1997) Effects of apomorphine on globus pallidus neurons in parkinsonian patients. Ann Neurol 42:767-775.

Jenner P (2008) Molecular mechanisms of L-DOPA-induced dyskinesia. Nat Rev Neurosci 9:665-677.

Keifman E, Ruiz-DeDiego I, Pafundo DE, Paz RM, Solís O, Murer MG, Moratalla R (2019) Optostimulation of striatonigral terminals in substantia nigra induces dyskinesia that increases after L-DOPA in a mouse model of Parkinson's disease. Br J Pharmacol 176:2146-2161.

Kita H, Kita T (2011) Cortical stimulation evokes abnormal responses in the dopamine-depleted rat basal ganglia. J Neurosci 31:10311-10322.

Kita H, Nambu A, Kaneda K, Tachibana Y, Takada M (2004) Role of ionotropic glutamatergic and GABAergic inputs on the firing activity of neurons in the external pallidum in awake monkeys. J Neurophysiol 92:3069-3084

Legéndy CR, Salcman M (1985) Bursts and recurrences of bursts in the spike trains of spontaneously active striate cortex neurons. J Neurophysiol 53:926-939.

Levy R, Dostrovsky JO, Lang AE, Sime E, Hutchison WD, Lozano AM (2001) Effects of apomorphine on subthalamic nucleus and globus pallidus internus neurons in patients with Parkinson's disease. J Neurophysiol 86:249-260.

Lozano AM, Lang AE, Levy R, Hutchison W, Dostrovsky J (2000) Neuronal recordings in Parkinson's disease patients with dyskinesias induced by apomorphine. Ann Neurol 47:S141-S146.

Mallet N, Ballion B, Moine CL, Gonon F (2006) Cortical inputs and GABA interneurons imbalance projection neurons in the striatum of parkinsonian rats. J Neurosci 26:3875-3884

Maurice N, Deniau JM, Glowinski J, Thierry AM (1999) Relationships between the prefrontal cortex and the basal ganglia in the rat: physiology of the cortico-nigral circuits. J Neurosci 19:4674-4681.

Meissner W, Ravenscroft P, Reese R, Harnack D, Morgenstern R, Kupsch A, Klitgaard H, Bioulac B, Gross CE, Bezard E, Boraud T (2006) Increased slow oscillatory activity in substantia nigra pars reticulata triggers abnormal involuntary movements in the 6-OHDA-lesioned rat in the presence of excessive extracellular striatal dopamine. Neurobiol Dis 22:586-598.

Meredith GE, Kang UJ (2006) Behavioral models of Parkinson's disease in rodents: a new look at an old problem. Mov Disord 21:1595-1606.

Nambu A, Tokuno H, Hamada I, Kita H, Imanishi M, Akazawa T, Ikeuchi Y, Hasegawa N (2000) Excitatory cortical inputs to pallidal neurons via the subthalamic nucleus in the monkey. J Neurophysiol 84:289300

Nambu A, Tokuno H, Takada M (2002) Functional significance of the cortico-subthalamo-pallidal "hyperdirect" pathway. Neurosci Res 43:111117.
Nambu A, Tachibana Y, Chiken S (2015) Cause of parkinsonian symptoms: firing rate, firing pattern or dynamic activity changes? Basal Ganglia 5:1-6.

Nishibayashi H, Ogura M, Kakishita K, Tanaka S, Tachibana Y, Nambu A, Kita H, Itakura T (2011) Cortically evoked responses of human pallidal neurons recorded during stereotactic neurosurgery. Mov Disord 26:469476.

Nishijima H, Suzuki S, Kon T, Funamizu Y, Ueno T, Haga R, Suzuki C, Arai A, Kimura T, Suzuki C, Meguro R, Miki Y, Yamada J, Migita K, Ichinohe N, Ueno S, Baba M, Tomiyama M (2014) Morphologic changes of dendritic spines of striatal neurons in the levodopa-induced dyskinesia model. Mov Disord 29:336-343.

Obeso JA, Olanow CW, Nutt JG (2000a) Levodopa motor complications in Parkinson's disease. Trends Neurosci 23:S2-S7.

Obeso JA, Rodríguez-Oroz MC, Rodríguez M, Lanciego JL, Artieda J, Gonzalo N, Olanow CW (2000b) Pathophysiology of the basal ganglia in Parkinson's disease. Trends Neurosci 23:S8-S19.

Papa SM, Desimone R, Fiorani M, Oldfield EH (1999) Internal globus pallidus discharge is nearly suppressed during levodopa-induced dyskinesias. Ann Neurol 46:732-738.

Parker JG, Marshall JD, Ahanonu B, Wu Y-W, Kim TH, Grewe BF, Zhang Y, Li JZ, Ding JB, Ehlers MD, Schnitzer MJ (2018) Diametric neural ensemble dynamics in parkinsonian and dyskinetic states. Nature 557:177-182.

Perez XA, Zhang D, Bordia T, Quik M (2017) Striatal D1 medium spiny neuron activation induces dyskinesias in parkinsonian mice. Mov Disord 32:538-548.

Picconi B, Centonze D, Håkansson K, Bernardi G, Greengard P, Fisone G, Cenci MA, Calabresi P (2003) Loss of bidirectional striatal synaptic plasticity in L-DOPA-induced dyskinesia. Nat Neurosci 6:501506.

Picconi B, Bagetta V, Ghiglieri V, Paillè V, Di Filippo M, Pendolino V, Tozzi A, Giampà C, Fusco FR, Sgobio C, Calabresi $\mathrm{P}$ (2011) Inhibition of phosphodiesterases rescues striatal long-term depression and reduces levodopa-induced dyskinesia. Brain J Neurol 134:375-387.

Ryan LJ, Clark KB (1991) The role of the subthalamic nucleus in the response of globus pallidus neurons to stimulation of the prelimbic and agranular frontal cortices in rats. Exp Brain Res 86:641-651.

Ryan MB, Bair-Marshall C, Nelson AB (2018) Aberrant striatal activity in parkinsonism and levodopa-induced dyskinesia. Cell Rep 23:3438-3446. e5.

Sano H, Nambu A (2019) The effects of zonisamide on L-DOPA-induced dyskinesia in Parkinson's disease model mice. Neurochem Int 124:171180.

Sano H, Chiken S, Hikida T, Kobayashi K, Nambu A (2013) Signals through the striatopallidal indirect pathway stop movements by phasic excitation in the substantia nigra. J Neurosci 33:7583-7594.

Sano H, Murata M, Nambu A (2015) Zonisamide reduces nigrostriatal dopaminergic neurodegeneration in a genetic model of Parkinson's disease. J Neurochem 134:371-381.

Schallert T, Fleming SM, Leasure JL, Tillerson JL, Bland ST (2000) CNS plasticity and assessment of forelimb sensorimotor outcome in unilateral rat models of stroke, cortical ablation, parkinsonism and spinal cord injury. Neuropharmacology 39:777-787.

Suárez LM, Solís O, Caramés JM, Taravini IR, Solís JM, Murer MG, Moratalla R (2014) L-DOPA treatment selectively restores spine density in dopamine receptor D2-expressing projection neurons in dyskinetic mice. Biol Psychiatry 75:711-722.

Suárez LM, Solis O, Aguado C, Lujan R, Moratalla R (2016) L-DOPA oppositely regulates synaptic strength and spine morphology in D1 and D2 striatal projection neurons in dyskinesia. Cereb Cortex 26:42534264.

Tachibana Y, Kita H, Chiken S, Takada M, Nambu A (2008) Motor cortical control of internal pallidal activity through glutamatergic and GABAergic inputs in awake monkeys. Eur J Neurosci 27:238253.

Tachibana Y, Iwamuro H, Kita H, Takada M, Nambu A (2011) Subthalamopallidal interactions underlying parkinsonian neuronal oscillations in the primate basal ganglia. Eur J Neurosci 34:1470-1484.

Thiele SL, Warre R, Khademullah CS, Fahana N, Lo C, Lam D, Talwar S, Johnston TH, Brotchie JM, Nash JE (2011) Generation of a model of L- 
DOPA-induced dyskinesia in two different mouse strains. J Neurosci Methods 197:193-208.

Tillerson JL, Cohen AD, Philhower J, Miller GW, Zigmond MJ, Schallert T (2001) Forced limb-use effects on the behavioral and neurochemical effects of 6-hydroxydopamine. J Neurosci 21:4427-4435.

Walters JR, Hu D, Itoga CA, Parr-Brownlie LC, Bergstrom DA (2007) Phase relationships support a role for coordinated activity in the indirect pathway in organizing slow oscillations in basal ganglia output after loss of dopamine. Neuroscience 144:762-776.

Whalen TC, Willard AM, Rubin JE, Gittis AH (2020) Delta oscillations are a robust biomarker of dopamine depletion severity and motor dysfunction in awake mice. J Neurophysiol 124:312-329.
Wichmann T, Soares J (2006) Neuronal firing before and after burst discharges in the monkey basal ganglia is predictably patterned in the normal state and altered in parkinsonism. J Neurophysiol 95:2120-2133.

Wichmann T, Bergman H, Starr PA, Subramanian T, Watts RL, DeLong MR (1999) Comparison of MPTP-induced changes in spontaneous neuronal discharge in the internal pallidal segment and in the substantia nigra pars reticulata in primates. Exp Brain Res 125:397-409.

Yamamoto N, Pierce RC, Soghomonian J-J (2006) Subchronic administration of L-DOPA to adult rats with a unilateral 6-hydroxydopamine lesion of dopamine neurons results in a sensitization of enhanced GABA release in the substantia nigra, pars reticulata. Brain Res 1123:196-200. 